\title{
Cortical Networks for Recognition of Speech with Simultaneous Talkers
}

Christian Herrera, ${ }^{1}$ Nicole Whittle, ${ }^{1}$ Marjorie R. Leek,${ }^{1,2}$ Christian Brodbeck, ${ }^{3}$ Grace Lee, ${ }^{2}$ Caleb

Barcenas, ${ }^{2}$ Samuel Barnes, ${ }^{2}$ Barbara Holshouser, ${ }^{2}$ Alex Yi, ${ }^{1,2}$ and Jonathan H. Venezia ${ }^{1,2}$

1 VA Loma Linda Healthcare System, Loma Linda, CA

${ }^{2}$ Loma Linda University, Loma Linda, CA

${ }^{3}$ University of Connecticut, Storrs, CT

Corresponding Author:

Jonathan $\mathrm{H}$. Venezia

VA Loma Linda Healthcare System

Mail Code 151

11201 Benton Street

Loma Linda, CA 92357

(909) 825-7084 x7292

jonathan.venezia@va.gov 


\section{HIGHLIGHTS}

- Twenty-five listeners complete a 3-AFC speech recognition task with two simultaneous talkers during fMRI scanning; the two talkers utter identical phrases ("Unison" condition) or phrases that differ in terms of several key words ("Competing" condition)

- A spectrotemporal modulation filtering procedure is used together with spectrotemporal receptive field (STRF) modeling

- STRF models generate cross-validated predictions of brain activity that differ significantly between conditions in three cortical networks: a bilateral superior temporal Intelligibility network, a frontoparietal Distortion network, and a Semantic network spanning canonical semantic memory regions

- The Intelligibility network responds primarily to intelligible speech, is positively associated with speech recognition performance, and is entirely STRF mediated; the Distortion network responds to acoustic and non-acoustic degradation of target speech, is negatively associated with speech recognition performance, and is only partially STRF mediated; the Semantic network responds to intelligible speech, is positively associated with speech recognition performance, and is only partially STRF mediated

- The Intelligibility and Distortion networks make partially independent and roughly equalmagnitude contributions to speech recognition performance, and these contributions entirely supersede those of the Semantic network, suggesting a dynamic interaction between bottom-up/non-task-dependent networks (Intelligibility) and top-down/task-dependent networks (Distortion/Semantic) in support of speech recognition with and without a competing talker 
The relative contributions of superior temporal (auditory) vs. inferior frontal and parietal (sensorimotor) networks to recognition of speech against competing speech remain unclear, although the contributions themselves are well established. Here, we use fMRI with spectrotemporal receptive field (STRF) modeling to examine the speech information represented in temporal vs. frontoparietal networks for two speech recognition tasks with and without a competing talker. We also generate 'neurometric functions' that describe the relative contributions of these networks to speech recognition performance. Specifically, 25 listeners completed two versions of a 3-Alternative Forced-Choice (3-AFC) competing speech task: "Unison" and "Competing", in which a female (target) and a male (competing) talker uttered identical or different phrases, respectively. Spectrotemporal modulation filtering was applied to the two-talker mixtures and a "boosting" procedure was used to generate STRF models to predict brain activation from differences in spectrotemporal distortion on each trial. STRF model predictive accuracy was better for Competing than Unison in a bilateral temporal lobe network, and better for Unison than Competing in a large network of frontoparietal and midline brain regions. Agglomerative STRF clustering further revealed three subnetworks: a bilateral superior temporal Intelligibility network, a frontoparietal Distortion network, and a Semantic network distributed across classic semantic memory regions. The Intelligibility and Semantic networks responded primarily to spectrotemporal cues associated with speech intelligibility, regardless of condition, while the Distortion network responded to the absence of such cues in both conditions, but also to the absence (presence) of target-talker (competing-talker) vocal pitch in the Competing condition, suggesting a generalized response to signal degradation. Neurometric function analysis showed that: (i) activation in the Intelligibility network was strongly positively correlated with behavioral performance and that this relation was entirely STRF-mediated; and (ii) activation in the Distortion network was strongly negatively correlated with performance and this relation was only partially STRF-mediated. The contributions to performance from these networks were partially independent and of roughly equal magnitude. Finally, activation in the Semantic network was weakly positively correlated with performance and this relation was entirely superseded by those in the Intelligibility and Distortion networks. We conclude: (a) superior temporal regions play a bottom-up, perceptual role in competing speech tasks; (b) frontoparietal regions play a top-down, task-dependent role in competing speech tasks that scales with listening effort; and (c) performance ultimately relies on dynamic interactions between these networks, with additional contributions from semantic regions that likely scale with the semantic predictability of the speech material.

Keywords: fMRI, spectrotemporal receptive fields, speech recognition, competing speech, auditory, sensorimotor 


\section{INTRODUCTION}

The classic model of speech processing in the brain, informed primarily by neuropsychological studies, is that receptive speech is mediated by sensory brain regions in the superior temporal lobe, while expressive speech is mediated primarily by motor brain regions in the inferior frontal lobe (Damasio and Geschwind, 1984; Hickok and Poeppel, 2000; Gernsbacher and Kaschak, 2003; Damasio et al., 2004). A prominent challenge to the classic view is the motor theory of speech perception, which posits that gestures - not speech sounds - are the objects of speech perception, thus implicating motor brain regions in receptive speech (Liberman, 1957; Liberman and Mattingly, 1985; Galantucci et al., 2006). Although the motor theory of speech perception was initially rebuked due to its failure to account for the classic neuropsychological findings, among other shortcomings (Diehl et al., 2004; Holt and Lotto, 2008; Lotto et al., 2009; Scott et al., 2009; Venezia and Hickok, 2009; Stokes et al., 2019), it experienced a major resurgence when modern, non-invasive brain imaging methods revealed unequivocally that inferior frontal brain regions are engaged during speech perception (Rizzolatti and Craighero, 2004; Pulvermuller and Fadiga, 2010; D'Ausilio et al., 2012). More recently, even sensory-oriented speech researchers have come to acknowledge that frontal motor regions play at least some role in speech perception, leading to a moderation of the classic model in which bottom-up sensory processing is subserved by superior temporal brain regions while inferior frontal regions contribute to top-down or task-sensitive aspects of speech perception (Binder et al., 2004; Hickok and Poeppel, 2007; Venezia et al., 2012). Broadly, speech researchers tend to endorse one of two views regarding the specific contribution of the inferior frontal brain regions during receptive speech. One proposes a "frontal-motor" mechanism, whereby motor speech circuits generate sensory predictions via "efference copy" that constrain the perceptual interpretation of incoming speech sounds (Skipper et al., 2007; Bever and Poeppel, 2010; Schwartz et al., 2012; Cope et al., 2017), while the other proposes a "frontal-cognitive" mechanism, whereby inferior frontal regions are engaged by domain general or "multiple-demand" processes required by receptive speech tasks (Blank et al., 2014; Noyce et al., 2017; Mineroff et al., 2018; Diachek et al., 2020). Here, an area of possible convergence is phonological working memory, which has been shown to rely on speech production circuits in the inferior frontal lobe but also contributes to a range of tasks that spans beyond speech perception or recognition per se (Jacquemot and Scott, 2006; Buchsbaum et al., 2011; Scott and Perrachione, 2019).

Frontal brain circuits may be especially important for recognition of degraded speech or speech in background noise. Indeed, this notion has been embraced by motor theorists, with recent variants of the motor theory suggesting that top-down motor predictions figure most prominently in speech perception when the input signal is corrupted or otherwise ambiguous (Stokes et al., 2019), and by the nascent field of cognitive hearing science, which posits a significant role for domain general or cognitive systems when speech is either intrinsically (e.g., with hearing loss) or extrinsically (e.g., with background noise) distorted (Arlinger et al., 2009; Rönnberg et al., 2011; Pichora-Fuller et al., 2016). Notably, while these perspectives differ in terms of their emphasis on frontal-motor versus frontal-cognitive mechanisms, they fundamentally agree that frontal contributions scale with the demands of the task and thus do not constitute a core component of bottom-up speech perception. Numerous recent imaging studies show that while both superior temporal and inferior frontal regions are recruited during listening tasks with noisy 
or degraded speech, the response in frontal regions is compensatory while the response in temporal regions is obligatory. For example, a classic finding is that temporal regions respond more strongly when speech is less degraded and/or recognition performance is better, while frontal regions respond more strongly when speech is more degraded, recognition performance is worse, and/or when attention is directed to the speech stimulus (Wong et al., 2008, 2009; Mattys et al., 2012; Wild et al., 2012; Erb and Obleser, 2013; Erb et al., 2013; Vaden et al., 2013, 2015, 2016, 2017; Du et al., 2016). Evidence from recent transcranial magnetic stimulation studies further shows that disruption of either left inferior frontal or left/right superior temporal regions impairs speech perception in noise (David et al., 2007; Venezia et al., 2019b; Kennedy-Higgins et al., 2020); however, inferior frontal stimulation has only been shown to disrupt sublexical speech perception, while superior temporal stimulation has been shown to disrupt comprehension of continuous speech, a crucial distinction which again supports a

With respect to the relative contributions of temporal and frontal brain regions to speech recognition in background noise, an area of particular interest is recognition of speech in scenarios with multiple simultaneous talkers (the "cocktail party" phenomenon; Cherry, 1953). Here, existing data show that the target speech stream is represented selectively in both temporal and frontal/motor regions, and the background or "competing" speech stream may also be represented selectively in the frontal cortex (Golumbic et al., 2013; Rimmele et al., 2015; Gao et al., 2017; Puschmann et al., 2017, 2019; Fiedler et al., 2019). These findings suggest frontal mechanisms may play an outsize role in speech recognition with multiple competing talkers. The purpose of the present study is to determine, for a scenario with two simultaneous talkers, exactly what speech information is encoded in temporal versus frontal brain regions, and what relative contributions these mechanisms make to behavioral performance on a competing speech task. To this end, we use a recently developed functional magnetic resonance imaging (fMRI) paradigm for estimating speech-driven spectrotemporal receptive fields (STRFs) called Auditory Bubbles (Venezia, 2019). Briefly, Auditory Bubbles works by applying a spectrotemporal modulation filter to a speech signal - typically a sentence - such that some spectrotemporal patterns are retained in the signal and others are removed. Different filter patterns are generated quasi-randomly from trial to trial, and the filter patterns themselves - each reflecting the underlying pattern of spectrotemporal modulation features in the speech signal - are then used to predict the brain response. At a given location in the brain, the extent to which a given spectrotemporal modulation feature contributes to the predicted brain signal becomes that feature's 'weight,' whereas the pattern of weights across all spectrotemporal modulation features is that brain region's STRF. Thus, Auditory Bubbles yields two important measures: (1) the ability of the STRF to predict the brain response, and (2) the STRF itself. Moreover, since spectrotemporal modulation filtering distorts the speech signal and modulates intelligibility, we can obtain a 'behavioral STRF' for the task at hand by determining the extent to which different spectrotemporal features contribute to speech recognition performance.

Here, we employ a variant of the Coordinate Response Measure (CRM; Bolia et al., 2000a) - a matrix-style competing speech test - with target female and competing male talkers (see (Venezia et al., 2020). The male and female talkers are generated synthetically from the same 
original waveforms using a vocal gender-morph procedure, thus rendering the talkers identical other than their vocal gender characteristics. Crucially, different spectrotemporal modulation features capture male versus female vocal pitch, yielding a STRF feature space with three important regions: (1) female (target) vocal pitch; (2) male (competing) vocal pitch; and (3) shared phonetic content across both talkers (Figure 1). Moreover, since the talkers' voices were generated synthetically from the same underlying waveforms, we can allow each talker to generate the exact same utterance in perfect synchrony. Thus, we employ two conditions: Competing, in which the female and male talkers generate similar utterances that differ by several key words (in classic matrix-speech-test style), and Unison, in which the female and male talkers generate the exact same utterance (essentially a "choral" talker in a quiet background). For each condition, we estimate neural STRFs and use them to predict the brain response at individual voxels throughout the cortex. The crucial comparison is whether the predictions pattern as "Competing > Unison" or "Unison > Competing." By examining the different networks identified by these contrasts, the different STRF patterns within those networks, and the correlation of each network's activity with performance on the CRM task, we hoped to clarify the relative contributions of temporal and frontal lobe brain regions to recognition of speech with multiple simultaneous talkers. 

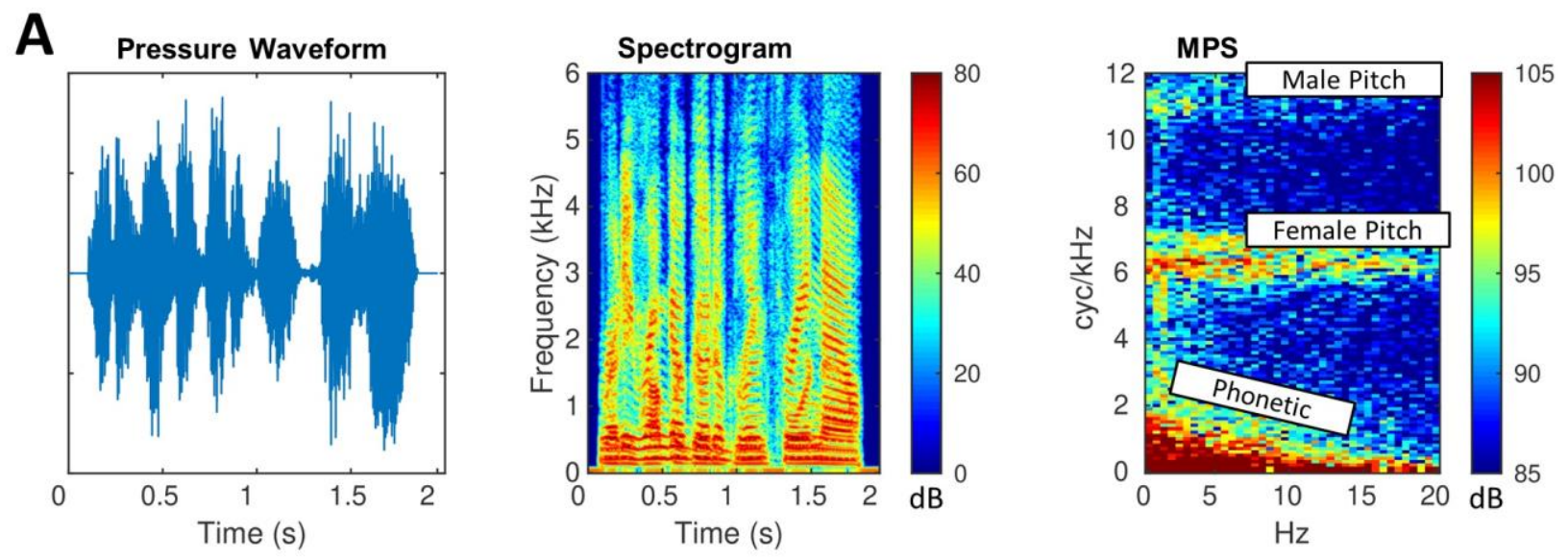

B
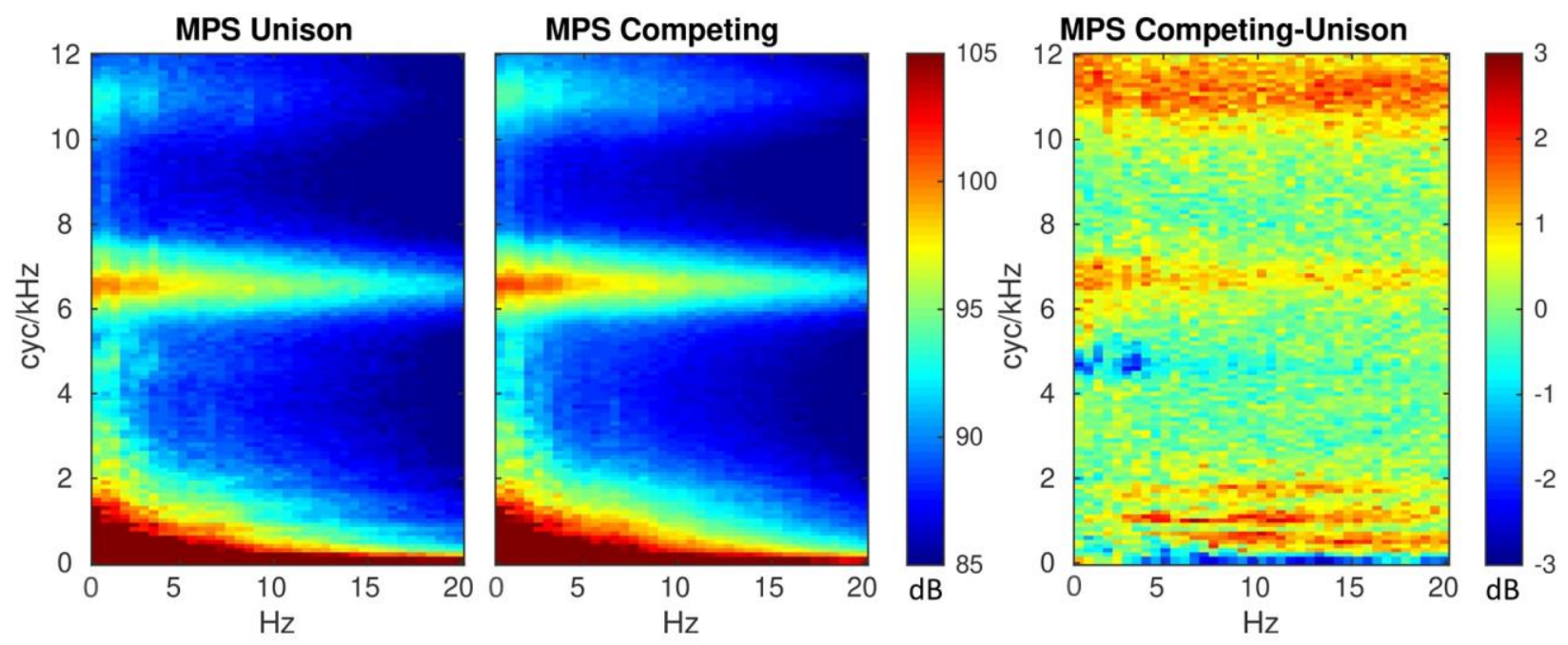

Figure 1. (A) Example of a single CRM item in the Competing condition. The pressure waveform (left panel) is a mixture of two CRM sentences spoken by male and female talkers whose vocal characteristics were generated synthetically from the same underlying recordings (originally male). STRFs are obtained in the domain of the modulation power spectrum (MPS; right panel), which is obtained as the 2D Fourier transform of a log-magnitude spectrogram with linear frequency spacing (middle panel). Speech energy occupies three regions divided along the ordinate of the MPS: phonetic content (0-2 cyc/k $\mathrm{Hz}$ ), female-taker pitch (5-7 cyc/kHz), and male-talker pitch (10-12 $\mathrm{cyc} / \mathrm{kHz}$ ). (B) Average MPS over 400 randomly generated CRM items in the Competing (left panel) and Unison (middle panel) conditions. The difference (Competing minus Unison) between conditions (right panel) reflects modest "unmasking" of speech energy in all three regions of the MPS in the Competing condition, but these differences were $<=3 \mathrm{~dB}$. That is, the conditions were well matched for overall speech energy in the MPS domain.

As we discuss below, our results support the conclusions that: (a) superior temporal regions play a bottom-up, perceptual role in competing speech perception; (b) inferior frontal and other "non-auditory" regions play a top-down, task-dependent role in competing speech perception; and (c) performance on a competing speech task ultimately relies heavily on both (bottom-up and top-down) networks. 


\section{MATERIALS AND METHODS}

\subsection{Participants}

\subsubsection{Recruitment}

Participants were recruited via digital advertisements posted in the VA Loma Linda Healthcare System (VALLHCS) main hospital and Ambulatory Care Center, word of mouth, VA medical record search, or from a contact database maintained by the Auditory Research Laboratory at VALLHCS.

\subsubsection{Inclusion Criteria}

Participants were Veterans of the United States Armed Forces between the ages of 18 and 60 years with a history of overseas deployment. There were no restrictions based on gender, ethnicity, or race. A preliminary screening was performed via phone and/or medical record review to verify: (a) age 18-60 years, (b) history of overseas deployment, (c) no history of conductive audiologic pathology including ear infections or ear surgeries, (d) English as a first language, and (e) no history of a major neurological disorder such as stroke, tumor, epilepsy, or moderate-to-severe traumatic brain injury. A secondary screening and audiologic evaluation was conducted in person to verify: (a) pure-tone average (PTA) air conduction thresholds (0.5, 1,2 , and $4 \mathrm{kHz}$ ) of $35 \mathrm{dBHL}$ or better in each ear, (b) pure-tone air conduction thresholds no worse than $40 \mathrm{dBHL}$ at any two audiometric frequencies below $8 \mathrm{kHz}$, (c) no differences in puretone thresholds exceeding $10 \mathrm{dBHL}$ between the two ears at more than one audiometric frequency below $4 \mathrm{kHz}$, (d) no evidence of conductive pathology, and (e) a score of at least 25 on the Mini-Mental State Examination (MMSE; Folstein et al., 1975). Participants were not excluded for active/historical psychiatric diagnosis or active/historical use of psychotropic medications, so long as these did not interfere substantially with performance of the experimental tasks. Since these data were collected as part of a larger study examining the links between aging and blast exposure, participants were also screened for exposure to highintensity blasts (e.g., artillery, mortar, plastic explosive, improvised explosive device) during their military service using a modified version of the Quantification of Cumulative Blast Exposure (QCuBE; Petrie et al., 2014) and enrolled as either "blast exposed" or "unexposed." All participants enrolled as blast exposed had self-reported difficulty understanding speech in background noise. Blast exposure status was not directly relevant to the present study, although individual differences potentially related to blast exposure were addressed in the analysis (see Sections 2.3.2, 2.3.5, Supplementary Information).

\subsubsection{Sample Characteristics}

A total of 25 Veterans (22 male, 3 female) participated in this study. The average age of the participants was 49.4 years (SD $=9.2$ years, range $=30-60$ years). Five of the participants were under age 40 , and of these four were blast exposed. Of the remaining 20 participants between ages 40-60, six were blast exposed. Among the participants, 16 had clinically significant PTSD symptoms (via medical record review or the PTSD checklist - military version; (Wilkins et al., 2011), 8 had a history of mild traumatic brain injury (medical record review or QCuBE), and 16 were diagnosed with another psychiatric disorder such as depression, anxiety, or substance use disorder (medical record review). One participant reported experiencing momentary lapses in consciousness, but no history of epilepsy or sleep disorder was identified in the participant's 
medical record. Another participant was found to have a small lesion spanning portions of the left medial temporal and occipital cortices. The participant did not report a history of stroke upon enrollment, so this was discovered only upon clinical review of the participant's structural MRI. Since this participant experienced no gross deficits in perception or cognition, the participant's data were retained in the sample.

\subsubsection{Study Administration and Informed Consent}

All study procedures were completed at the VALLHCS Auditory Research Lab or at the Loma Linda University Medical Center (LLUMC) East Campus Radiology Department. Prior to enrollment in the study, all participants provided their written informed consent. All participants received monetary compensation for their participation. All study documentation and procedures were reviewed and approved by the VALLHCS Institutional Review Board. The study was also registered with the LLUMC Institutional Review Board, which deferred oversight to VALLHCS.

\subsection{Procedures}

\subsubsection{Audiologic Evaluation}

Pure-tone thresholds were assessed using a Madsen Astera2 audiometer (Natus, Inc.) and modified descending Hughson-Westlake procedure at $0.25,5,1,2,3,4,6$, and $8 \mathrm{kHz}$ with ER$3 \mathrm{~A}$ insert earphones for air conduction and at $0.25,5,1,2,3$, and $4 \mathrm{kHz}$ with bone oscillator for bone conduction. Otoscopy, middle ear immittance testing (Tympstar, Grason-Stadler, Inc.) including tympanometry and ipsilateral acoustic reflex testing at $1 \mathrm{kHz}$, and bone conduction thresholds were used to rule out conductive pathology.

\subsubsection{Behavioral Test Battery and Questionnaires}

Participants completed an extensive behavioral test battery including assessments of speech perception in noise, auditory temporal processing, working memory, executive function, and processing speed; in addition, they completed a number of questionnaires to assess subjective hearing, mental health status, substance use, and military service history. These measures were entered as nuisance covariates in the second-level functional imaging analysis (2.3.5) after dimension reduction via principal components analysis (2.3.2). A detailed description of the behavioral test battery is provided in the Supplementary Information.

\subsubsection{Functional Magnetic Resonance Imaging}

\subsubsection{Speech Stimuli}

The source audio files (. wav; sampling rate $=20 \mathrm{kHz}$; quantization $=16$-bit) were sentences from the Coordinate Response Measure (CRM) corpus (Bolia et al., 2000) spoken by a single male talker (Talker 0). Using a vocal-gender shifting procedure described by Venezia et al. (2020), two versions of the CRM sentences were derived from the source files: (1) sentences spoken by a synthetic female talker with mean fundamental frequency scaled by 1.7 and vocal tract length scaled by 0.84 relative to the original 'Talker 0' values; and (2) sentences spoken by a male talker processed through the same gender-shift algorithm but with the mean fundamental frequency and vocal tract length each scaled by 1.0 relative to the original 'Talker 0 ' values. Gender shifting was performed using the pitch-synchronous overlap-add (PSOLA) procedure as implemented in PRAAT (Boersma, 2001). This process ensured the male and 
synthetic female talkers would differ only in terms of mean fundamental frequency (pitch) and vocal tract length, but not in terms of other talker-specific speech patterns that could produce differences in the MPS (e.g., speech rate or stress patterns).

The stimuli for the fMRI experiment were two-talker mixtures consisting of one CRM sentence spoken by the synthetic female talker and one CRM sentence spoken by the male talker. The CRM sentences take the form "Ready [call sign] go to [color] [number] now." There are eight possible call signs ("arrow", "baron”, "charlie”, "eagle”, "hopper”, “laker", "ringo”, "tiger”), four possible colors ("blue”, "green”, "red”, "white”), and eight possible numbers (1-8), yielding 256 total sentences comprising the factorial combinations of call sign, color and number. Two types of two-talker mixtures denoted "Competing" and "Unison" were generated as follows. To generate the Competing mixtures, a target sentence spoken by the synthetic female talker was chosen by selecting one of the 256 CRM sentences at random. A competing sentence spoken by the male talker was selected quasi-randomly such that the call sign, color, and number were different from the target sentence. The relative levels of the target and competing sentences were then adjusted to produce $\mathrm{a}+3 \mathrm{~dB}$ target-to-masker ratio (TMR). The Unison mixtures were generated in the same way except that the "competing" sentence spoken by the male talker was chosen to have the same call sign, color, and number as the target sentence. Since the synthetic female and male sentences were derived from the same underlying source waveforms, the Unison mixtures therefore comprised synthetic female and male voices uttering the exact same words in perfect synchrony. Perceptually, this is akin to listening to a single utterance in a quiet background, but as shown in Figure 1B the Unison mixtures retain the same broad pattern of speech energy in the MPS domain (including harmonics/vocal pitch from both talkers) as the Competing mixtures.

For both Unison and Competing mixtures, the male and synthetic female voices occupy discrete regions of the MPS along the spectral modulation dimension. The synthetic female voice appears at lower spectral modulation rates (higher pitch; 5-7.5 cyc/kHz) and the male voice appears at higher spectral modulation rates (lower pitch; 10-12.5 cyc/kHz). Formant-scale spectral components - here broadly termed "phonetic content" - appear at the lowest spectral modulation rates $(<2.5 \mathrm{cyc} / \mathrm{kHz})$ and cannot be uniquely attributed to the male talker or synthetic female talker (i.e., the talkers overlap in the MPS region representing phonetic content). Though the Unison and Competing mixtures are broadly similar as represented on the MPS (Figure 1B, left panels), there are some subtle differences in long-term modulation energy between mixture types (Figure 1B, right panel). Most notably, the Competing mixtures contain more modulation energy in the MPS regions representing the male talker's voice, the synthetic female talker's voice, and the shared phonetic content at temporal modulation rates greater than $3 \mathrm{~Hz}$. Competing mixtures contain more vocal energy for each talker relative to Unison mixtures because, in the Competing case, the talkers' signals are temporally non-overlapping (at least partially, versus completely overlapping in the Unison case), which leads to a greater number of acoustic "frames" with vocal energy from a single talker in isolation (i.e., an "unmasking" of vocal energy). The unmasking effect is larger for the male talker because the synthetic female talker has more vocal energy at the $+3 \mathrm{~dB}$ TMR. Competing mixtures contain more modulation energy for phonetic content above $3 \mathrm{~Hz}$ again because the talkers' signals are temporally non- 
overlapping, which in this case leads to "filling" of temporal dips in the speech signal of one talker with speech content from the other talker and, thus, appearance of speech energy at higher temporal modulation rates when considering the entire mixture. Although these effects are clearly present in the long-term MPS patterns, they are relatively small (no greater than a 3 $\mathrm{dB}$ difference in Unison vs. Competing; Figure 1B, right panel).

To generate the final stimuli for the fMRI experiment, bubbles filtering was applied to the Unison and Competing mixtures in the MPS domain as follows (steps 1-2 can be visualized in Figure $1 \mathrm{~A}$ and steps 3-5 can be visualized in Figure 2A): (1) obtain a log-magnitude spectrogram using Gaussian windows; (2) obtain the MPS by two-dimensional Fourier transform (2D-FFT) of the spectrogram; (3) retain randomly-selected segments of the MPS while removing the remainder by applying a binary, multiplicative bubbles filter; (4) obtain a filtered magnitude spectrogram by inverse 2D-FFT; and (5) obtain a filtered waveform by inverting the magnitude spectrogram using phase gradient heap integration (Prǔša and Balazs, 2017). Prior to filtering, waveforms were zero-padded to a fixed duration to ensure equal MPS dimensions (in pixels) across items. Bubbles filters were applied to the MPS region representing spectral modulations $\leq 15 \mathrm{cyc} / \mathrm{kHz}$ and temporal modulations $\leq 50 \mathrm{~Hz}$. Each bubbles filter was created by generating an all-zero image of dimensions equal to this MPS region, setting a randomly-selected subset of pixels (equal to the number of bubbles) to a value of one, smoothing with a 2D Gaussian filter with standard deviation of $0.5 \mathrm{cyc} / \mathrm{kHz}$ in the spectral modulation dimension and $2 \mathrm{~Hz}$ in the temporal modulation dimension, and binarizing the resulting image using a cutoff value of 0.1 . For a given CRM mixture, the bubbles filtering procedure took less than a second on our hardware, so filtering was performed in "real time" during the experiment. The final stimuli presented to one example participant can be found at https://osf.io/ftmpa/ in the Files repository 


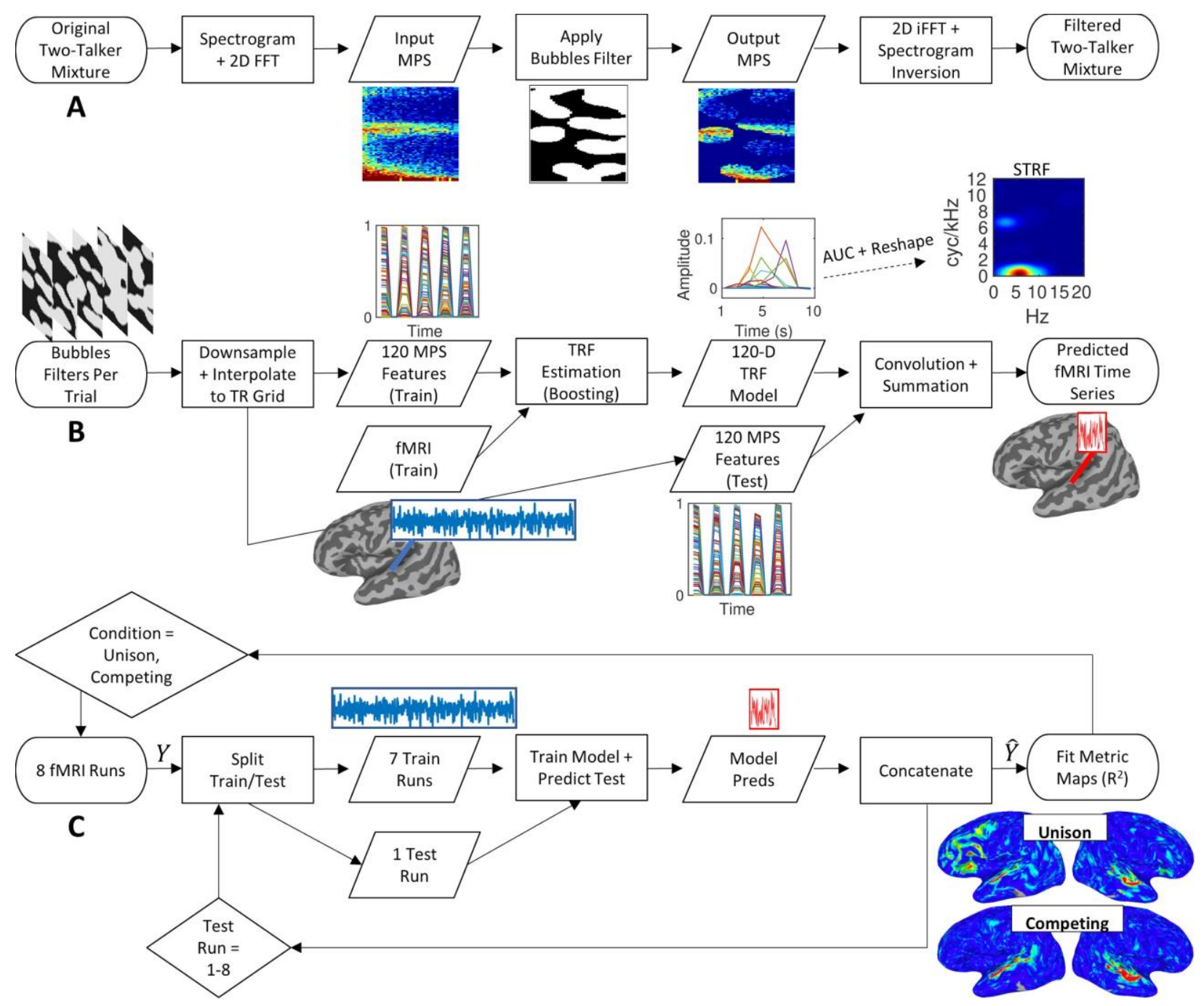

Figure 2. (A) Flow-chart schematic showing the procedure for generating a single bubbles-filtered CRM item. The bubbles filter is applied to the MPS of the two-talker mixture, resulting in a filtered waveform which retains only a subset of the MPS elements that were present in the original. The bubbles filter is retained for subsequent analysis. Separate, randomly-generated bubbles filters are applied to each item for each listener. (B) Flow-chart schematic showing the STRF fitting procedure at a single cortical surface node in a single listener. The bubbles filters applied to the stimulus on each trial are downsampled and interpolated to the TR grid producing 120 MPS feature vectors. These feature vectors are used to train a 120-D temporal response function (TRF) model using the boosting procedure, and the resultant model is used to predict held-out fMRI data. The STRF is obtained as the area under the curve of each TRF element. (C) Flow-chart schematic showing the nested cross-validation procedure used to produce STRF model prediction maps at the individual subject level. At each surface node, the fMRI data are split into training (7 runs) and test (1 run) sets. The procedure shown in ( $B$ ) is then applied (Train Model + Predict Test), where model training itself uses a hold-one-run-out cross-validation procedure on the 7 training runs (inner crossvalidation loop). This is repeated holding out each $\mathrm{fMRI}$ run as the test data (outer cross-validation loop). The model predictions from each iteration of the outer loop are concatenated and used to compute an overall model $R^{2}$, which is obtained separately at each surface node in each condition (Unison, Competing). 
The experimental task was a modified, three-alternative-forced-choice (3-AFC) version of the standard CRM task, which is to identify the color and number spoken by the target talker. In the standard task, there are 32 possible color/number combinations. In the present experiment, responses were speeded to allow more trials to be collected inside the scanner, which precluded the use of a 32-alternative response array. Instead, a 3-AFC task was employed in which the participant was asked to select the target color/number combination from among three choices displayed on the computer screen as filled squares (blue, green, red, or white) with a single digit in center (1-8; black font). The squares ( 3.5 degrees visual angle) were displayed in a horizontal row (each square separated by $\sim 5$ degrees visual angle) against a uniform gray background. One of the response alternatives was always the color/number spoken by the target (synthetic female) talker. For Competing mixtures, a second response alternative was always the color/number spoken by the competing (male) talker. For Unison mixtures, the second response alternative was generated as if a competing sentence had been spoken by the male talker (i.e., matching neither the color nor the number of the target sentence). For both Competing and Unison mixtures, a third response alternative was selected from one of five categories as follows: (1) same color as the first alternative; (2) same number as the first alternative; (3) same color as the second alternative; (4) same number as the second alternative; or (5) neither the same color nor the same number as the other two alternatives. The category of the third alternative was selected quasi-randomly such that each category was chosen an equal number of times in any given block of trials. The horizontal display location (left, center, right) of the three response alternatives was chosen at random.

In the scanner, trial initiation was synchronized to TR onset. On each trial, the auditory stimulus was presented $200 \mathrm{~ms}$ after TR onset. The stimulus duration was $1.96 \mathrm{~s}$. At stimulus onset, the words "Listen Now" were displayed in the center of the screen and they remained on screen until the end of the stimulus. At stimulus offset, "Listen Now" was replaced on screen with "Respond Now!" and the response array was displayed immediately beneath. The participant indicated their response with a button press. The response period was $2.5 \mathrm{~s}$. If a button press was recorded within the response period, "Respond Now!" was replaced with "Good," which remained onscreen for the remainder of the trial. If no button press was recorded, "Respond Now!" remained on screen until the end of the trial and the response was counted as incorrect. The subsequent trial was initiated at the onset of the next TR (140 ms after the end of the response period; total trial duration $=4.8 \mathrm{~s}$ ). Each participant completed 400 Competing trials and 400 Unison trials on separate days of scanning. The Competing trials were always completed on the first day and the Unison trials completed on the second day. One participant completed the Competing and Unison trials on the same day in separate scanning sessions separated by a break of about two hours. Most participants (18 of 25) completed the Competing and Unison scan sessions no more than two weeks apart. Another three participants completed the sessions no more than a month apart, and the remaining participants completed the sessions within $32,34,41$, and 115 days of one another.

On all trials, the auditory stimulus (CRM mixture) was processed with the bubbles filtering algorithm (i.e., the CRM task was performed in the presence of acoustic distortion imposed by 
bubbles). For Competing mixtures, the number of bubbles applied on each trial was determined using a weighted staircase procedure (Kaernbach, 1991) to target $66.67 \%$ correct performance (the midpoint of the psychometric function for 3-AFC). Two interleaved staircases were employed with the initial number of bubbles set to 50 and 150. Based on pilot data, these starting values tended to produce ascending and descending staircases, respectively, although threshold performance was close to or beyond one of the starting values for some listeners. To target $66.67 \%$ correct, the number of bubbles was decreased by 5 (more acoustic distortion) when the response was correct and increased by 10 (less acoustic distortion) when the response was incorrect. Participants completed a total of 400 Competing trials in blocks of 50 . The weighted staircases proceeded uninterrupted across all 400 trials. Ten additional TRs were collected at the end of each block and the scanner was stopped between blocks. Participants were offered the chance to rest between blocks (self-paced except for a mandatory rest at the halfway point) and were informed of the number of blocks completed at the end of each block.

For Unison mixtures, the number of bubbles was chosen to match exactly the trial-by-trial trajectory produced by the interleaved staircases employed in the Competing trials. Thus, participants completed a total of 400 Unison trials in blocks of 50, just as for Competing trials, and the Unison trials were not adaptive; the Unison trials were always performed after the Competing trials, on a separate day of scanning, so that the trajectory of the Competing staircases would be known. This ensured that the overall amount of acoustic distortion introduced by bubbles filtering would be matched across the Unison and Competing conditions. Since the CRM task is intrinsically easier in the Unison condition, the weighted staircase procedure applied to the Competing condition would invariably - if applied to the Unison condition - introduce more acoustic distortion in order to drive performance to $66.67 \%$ correct. Conversely, performance in the Unison condition is invariably better than $66.67 \%$ correct using the predetermined staircase trajectories from the Competing condition. From our perspective, it was preferable to match the conditions acoustically (number of bubbles) rather than by task difficulty (percent-correct performance). It is important to note that, regardless of the number of bubbles on a given trial, the location of each bubble in the MPS was selected at random, which effectively produced a unique pattern of acoustic distortion on every trial. These locations were stored so that the unique bubbles filter presented on each trial could be reconstructed during off-line analysis.

At the scanner, speech signals were delivered diotically via an external sound card (Scarlett 2i2, Focusrite, Inc.), amplifier (AMP100VS, AudioSource, Inc.), and MRI-compatible insert earphones (Model S14, Sensimetrics, Inc.). For presentation, bubbles-filtered CRM mixtures were upsampled to $44100 \mathrm{~Hz}$, processed with a digital equalization filter provided by Sensimetrics to produce flat frequency response, attenuated to produce an overall presentation level of $85 \mathrm{~dB} \mathrm{SPL}$, and gated on and off with a 10-ms raised cosine ramp. Attenuation of scanner noise was achieved via isolating foam eartips (Comply Canal Tips, Hearing Components Inc.) and silicone-backed foam earmuffs (Hibermate, Inc.). Custom low-profile earmuffs (foam backed with engineered wood) were used for participants with a large head circumference. Visual stimuli were presented via an MRI-compatible digital display (SensaVue, Philips, Inc.) viewed on an angled-front-surface mirror mounted on the head coil. Responses 
were collected via a four-button MRI-compatible response pad (LS-LINE, Cedrus, Inc.) and button response unit (Lumina, Cedrus Inc.). The response pad was operated by the dominant hand. Control of the experiment including stimulus presentation, timing, scanner synchronization, and delivery of instructions was achieved via the Psychtoolbox v3 (Kleiner et al., 2007) within MATLAB (Mathworks, Inc.) on a Linux desktop PC (Meerkat, System76, Inc.) running Ubuntu v18.04 LTS (Canonical, Inc.).

Prior to each scan session, participants completed a practice session outside the scanner. The practice session included verbal and written instructions, a single CRM trial with no bubbles applied to the speech stimulus and an unlimited response period, ten additional CRM trials with no bubbles and a $2.5 \mathrm{~s}$ response period, and a block of 50 trials that was otherwise identical to the trial blocks performed at the scanner. During the practice trials, recorded MRI-scanner noise was played in the background to simulate the acoustic environment in the scanner. At the practice session, acoustic stimuli were delivered diotically via the internal sound card (C610/X99 chipset, Intel, Inc.) of a Linux desktop (Silverback Workstation, System76, Inc.) to insert earphones (ER-4, Etymotic, Inc.). Visual stimuli were displayed on an LCD monitor (VX2457MHD, ViewSonic, Inc.) and responses were collected via mechanical keyboard (Dell, Inc.). The practice trials were controlled via Psychtoolbox v3 running in MATLAB R2018A on Ubuntu v16.04 LTS.

\subsubsection{Image Acquisition}

Images were acquired on a 3T MAGNETOM Skyra (Siemens, Inc.) with a 32-channel head coil located at the Loma Linda University East Campus Radiology Center. At each scan session, an anatomical scout, T1 anatomical volume, field map scan, and eight runs of blood-oxygen-level dependent (BOLD) functional volumes were acquired in that order. The functional volumes were acquired with a simultaneous multi-slice echo-planar imaging (SMS-EPI) sequence without partial Fourier or in-plain acceleration (Smith et al., 2013) and with a 10\% slice gap to minimize interslice signal leakage (Cauley et al., 2014); there were 56 axial slices oriented parallel to the $\mathrm{AC}-\mathrm{PC}$ axis with interleaved slice order, multiband acceleration factor $=4, \mathrm{TR}=1.2 \mathrm{~s}, \mathrm{TE}=33$ $\mathrm{ms}$, flip angle $=45$ degrees, echo spacing $=0.61 \mathrm{~ms}$, phase encoding direction $=A P$, bandwidth $=2055 \mathrm{~Hz} / \mathrm{Px}$, voxel size $=2.5 \times 2.5 \times 2.75 \mathrm{~mm}$ (including $10 \%$ gap), and matrix $=84 \times 84$. In each functional run, 210 volumes were acquired to allow for $50 \mathrm{CRM}$ trials (four volumes per trial) plus 10 additional volumes to capture the hemodynamic response to the final trials. Thus, each scan session produced 1680 functional volumes (400 total CRM trials). The field map scan parameters were TR $=0.557 \mathrm{~s}, \mathrm{TE} 1=4.92 \mathrm{~ms}$, TE2 $=7.38 \mathrm{~ms}$, flip angle $=60$ degrees, and bandwidth $=510 \mathrm{~Hz} / \mathrm{Px}$, with the same slice prescription, slice thickness, voxel size, and matrix size as the functional volumes. The T1 anatomical volume was acquired with a magnetization prepared rapid gradient echo (MPRAGE) sequence with 176 sagittal slices, $\mathrm{TR}=1.95 \mathrm{~s}, \mathrm{TE}=$ $2.32 \mathrm{~ms}, \mathrm{TI}=917 \mathrm{~ms}$, flip angle $=8$ degrees, GRAPPA acceleration factor $=2$, bandwidth $=200$ $\mathrm{Hz} / \mathrm{Px}$, voxel size $=0.9 \times 0.938 \times 0.938 \mathrm{~mm}$, and matrix $=256 \times 256$. 


\subsection{Analysis}

\subsubsection{Behavior}

Performance on the in-scanner behavioral task was measured in two ways. First, the percentage of correct responses on the 3-AFC CRM task was calculated for Competing and Unison trials separately. For Competing trials, this was expected to yield $\sim 67 \%$ correct for all participants due to the weighted staircase procedure. For Unison trials, percent-correct performance was not constrained but was expected to exceed $67 \%$ for all participants. Second, the mean number of bubbles across all trials was tabulated only for Competing trials because this number was procedurally ensured to be the same for Unison trials. The mean number of bubbles indicates the amount of acoustic distortion a participant was able to tolerate, on average, to achieve $67 \%$ correct performance, where fewer bubbles indicate better performance (more acoustic distortion tolerated). To determine the effect of particular bubble patterns on performance, classification images ('behavioral STRFs') were calculated for each participant separately for Competing and Unison trials as follows:

$$
\text { CImg }=(1-P C) * \sum_{i}^{M} B u b_{i}+P C * \sum_{j}^{N} B u b_{j}
$$

where $i(j)$ is the trial index, $M$ is the number of correct trials, $N$ is the number of incorrect trials, $P C$ is the proportion of correct trials, and $B u b_{i(j)}$ is the $2 \mathrm{D}$ bubbles filter applied to the CRM mixture on a given trial. Whereas the bubbles filter is binary with ones indicating acoustic information retained in the MPS and zeroes indicating acoustic information removed from the MPS, positive values denote MPS regions that improved task performance and negative values denote MPS regions that impaired task performance. Second-level classification images were obtained for the Unison and Competing conditions by taking the mean across participants within each condition and smoothing the resultant image with a 2D Gaussian kernel (sigma $=3$ pixels).

\subsubsection{Nuisance Covariates: Principal Component Analysis}

Supplementary Table 1 describes a number of auxiliary variables collected outside the scanner that characterize the audiological status, speech-in-noise performance, auditory temporal acuity, subjective hearing, mental health, and cognitive abilities of the participants in this study. To generate a set of nuisance covariates summarizing individual differences that might impact the fMRI analysis, these variables were submitted to a principal component analysis. Only those components with eigenvalues greater than one were retained for further analysis. This yielded six components accounting for $80 \%$ of the total variance in the auxiliary data. For these six components, the principal component scores were obtained for each participant yielding six zero-centered nuisance covariates.

\subsubsection{MR Image Preprocessing}

Automated cortical surface reconstruction from the $\mathrm{T} 1$ anatomical volume collected during the Competing scan session was performed in Freesurfer v6.0 (http://surfer.nmr.mgh.harvard.edu). Right and left hemisphere cortical surface meshes were then converted to AFNI/SUMA (https://afni.nimh.nih.gov/Suma) format, co-registered to the native-space T1 volume, resampled to a standard topology via linear icosahedral tessellation with 64 edge divides, and merged into a single surface containing 81924 nodes using the prep_afni_surf.py function of the "surfing" toolbox v0.6 (https://github.com/nno/surfing; Oosterhof et al., 2011). For plotting, a standard topology surface mesh was also generated from the Colin 27 template brain. Using ANTs v 
3.0.0 (http://stnava.github.io/ANTs/), the Unison T1 volume was brought into alignment with the Competing $\mathrm{T} 1$ volume using a six-parameter rigid body transformation (antsRegsistrationSyn.sh). The obtained transformation matrix was later used to bring the preprocessed functional volumes from the Unison scan session into the space of the Competing scan session, so that the Unison data could be co-registered to the Competing T1 volume and mapped to the standard topology cortical surface.

The functional volumes from the Competing and Unison scan sessions were otherwise preprocessed identically. The volumes were converted to NIFTI format using dcm2niix v1.0.2 (https://github.com/rordenlab/dcm2niix) and slice-timing corrected in SPM12 (https://www.fil.ion.ucl.ac.uk/spm/software/spm12/) using the nii_SliceTime.m MATLAB script (https://www.mccauslandcenter.sc.edu/crnl/tools/stc) to automatically read the multiband slice times from the image header. Motion correction and unwarping via the field map scan were then performed in SPM12 via the CONN toolbox (RRID:SCR_009550 www.nitrc.org/projects/conn) release 18.b (Whitfield-Gabrieli and Nieto-Castanon, 2012). Automated tissue segmentation of the T1 volume was also performed in CONN. It was at this point that the Unison functional volumes were moved into the same space as the Competing data as described in the preceding paragraph. Co-registration to the Competing T1 volume was then performed in SPM12 via CONN using a 12-parameter affine transformation. Denoising was performed in CONN via regression with quadratic detrending and the following confounding variables: six parameter rigid body motion-correction parameters and their temporal derivatives, five principal components extracted from the signals within each of the white matter and cerebrospinal fluid regions of interest (ROIs) defined during tissue segmentation, and 'scrubbing' covariates determined from the CONN Artifact Rejection Tool (ART) with the 'liberal' setting to identify motion and signal spikes. Finally, the preprocessed functional data were mapped to the merged, standard-topology surface mesh and smoothed to a target level of $4 \mathrm{~mm}$ full width at half maximum using AFNI v19.0 (https://afni.nimh.nih.gov/).

\subsubsection{Spectrotemporal Receptive Field Modeling}

\subsubsection{Model Estimation}

The goal of STRF estimation was to produce a model that, given a sequence of 2D bubbles filters, would be able to predict the BOLD time series evoked by the sequence of CRM trials in which the acoustic signals were processed with those filters (Figure 2B). Independent models for the Competing and Unison conditions were estimated at each cortical surface node. That is, at a given node and for a given condition, the model determined whether and how a set of acoustic speech features - defined by the trial-by-trial patterns of information retained in the MPS after bubbles filtering - modulated the evoked BOLD signal. To generate a set of model predictors for a given participant, the set of 2D bubbles filters unique to that participant was downsampled, vectorized, and converted to a series of 'event timing' regressors. Specifically, each bubbles filter was cropped to the MPS region spanning $0-20 \mathrm{~Hz}$ and $0-12 \mathrm{cyc} / \mathrm{kHz}(121 \mathrm{x}$ 40 pixels; see 2.2.3.1), downsampled to $12 \times 10$ pixels using cubic interpolation, and vectorized to produce a 120-D acoustic feature space. A binary stimulus timing vector was then defined to take value one during stimulus presentation and zero otherwise; to this end, the acoustic stimuli presented during the experiment were regenerated offline and the onset and offset of sound 
energy for each trial were identified based on the windowed root-mean-square amplitude of the signal (silence threshold $=0.0035$ ). These measurements were used to generate a series of stimulus onsets and durations that defined the stimulus timing. The stimulus timing vector was initially generated at a sampling rate of $100 \mathrm{~Hz}$. To generate each of the final model predictors, the one-valued segments of the stimulus timing vector ('stimulus on') were replaced with the corresponding bubbles filter values, trial-by-trial, for a given feature in the 120-D feature space; the resultant predictors were then low-pass filtered at a cutoff frequency of $0.417 \mathrm{~Hz}$ with a Lanczos antialiasing filter and downsampled to the BOLD sampling rate of $0.833 \mathrm{~Hz}$ (de Heer et al., 2017). This produced a set of 120 predictors temporally aligned to the fMRI data and summarizing the trial-by-trial patterns of MPS features retained in the CRM mixtures after bubbles filtering.

To fit the model, the neural response at time $t, y_{t}$, was predicted from the sum of $N$ predictor variables, $x_{n}$, convolved with a corresponding response function, $h_{n}$, of length $T$ :

$$
\widehat{y_{t}}=\sum_{n}^{N} \sum_{\tau}^{T} h_{n, \tau} \cdot x_{n, t-\tau}
$$

The response function or 'kernel,' $h_{n}$, was estimated over time lags from $1.2 \mathrm{~s}$ to $9.6 \mathrm{~s}$ post stimulus onset using an iterative coordinate descent algorithm (David et al., 2007) as implemented by the eelbrain package v0.32 (DOI: 10.5281/zenodo.3923991) in Python v3.7.3 to minimize the $\ell 2$ error. Prior to model fitting, the neural signal, $y_{t}$, was low-pass filtered at a cutoff frequency of $0.15 \mathrm{~Hz}$ with a fourth-order Butterworth filter and normalized (z-score) separately within each functional run. The predictors, $x_{n}$, were also normalized (z-score) within each functional run.

\subsubsection{Model Predictions and Cross-Validation}

At a given cortical surface node, the neural response was predicted using nested, leave-onerun-out cross-validation (Figure $2 \mathrm{C}$ ). Within the Unison and Competing conditions separately, eight cross-validation folds were defined corresponding exactly to the eight runs of functional data collection. In an outer loop, the responses in each fold were predicted with kernels estimated from the remaining seven folds (Figure 2B). These predictions, combined, served to calculate the model performance defined as the proportion of variance in the true neural response explained by the predicted neural response $\left(R^{2}\right)$. In an inner loop (not pictured in Figure 2), each of the seven estimation folds was in turn used as the validation set for the kernel trained on the six remaining folds. Kernels were iteratively improved based on the maximum error reduction in the training set (the steepest coordinate descent) and validated in the validation set. Iteration stopped when the error for the training data could not be reduced any further, or when the error for the validation data increased in two successive iterations. When iteration stopped, the kernel from the iteration with the smallest error on the validation data was retained. The seven kernels thus produced by the inner loop were averaged and used to predict the neural response for the held-out fold of the outer loop (Eq. 1). The eight predicted time series corresponding to the held-out folds of the outer loop were concatenated in the original 
order and compared to the true neural response to calculate $R^{2}$ over the entire experiment, separately for the Unison and Competing conditions. This was repeated for each cortical surface node to produce node-wise maps of $R^{2}$ for Unison and Competing at the participant level.

\subsubsection{Spectrotemporal Receptive Fields}

The STRF at a given cortical surface node was obtained from the response kernel generated during model estimation. Kernels were first averaged over the eight folds of the outer crossvalidation loop. This yielded a kernel of dimension 120 (bubbles features) $\times 7$ (time steps, $T$, in Eq. 2). The kernel describes the hemodynamic response to each of the 120 bubbles features as an impulse response (Glover, 1999) though here the impulse responses were estimated jointly over the 120 features with sparseness (i.e., regularization) enforced by the iterative procedure (David et al., 2007). The kernel was converted to scalar values (STRF weights) by calculating the area under the curve (AUC) of the impulse response for each of the 120 features (Figure 2B). This was performed separately for each cortical surface node to produce 120-D STRF maps at the participant level (separately for Unison and Competing). To visualize STRFs at the group level, the STRF maps were averaged across participants and, for a given cortical surface node or region, the 120 group-averaged STRF weights were reshaped and upsampled to create a 2D array of the same dimensions as the original bubbles filters $(121 \times 40)$ and smoothed with a 2D Gaussian kernel (sigma $=3$ pixels).

\subsubsection{Second Level fMRI Analysis}

To visualize STRF model performance across participants, second-level $R^{2}$ maps were obtained by averaging the first-level $R^{2}$ maps across participants, separately for the Competing and Unison conditions. To identify cortical surface nodes for which STRF model predictions were significantly different between the Competing and Unison conditions, a first-level 'difference image' was obtained for each participant by subtracting the Unison $R^{2}$ map from the Competing $R^{2}$ map. These difference maps were then analyzed statistically at the second level using linear regression at each cortical surface node:

$$
d=\alpha+Z \gamma+\varepsilon
$$

where $d$ is the difference in $R^{2}$ between Competing and Unison, $\alpha$ is the intercept term, $Z$ is a matrix of zero-centered nuisance covariates (2.3.2), $\gamma$ is the vector of regression coefficients for the nuisance covariates, and $\varepsilon$ is the unexplained error. Here, the term of interest is the intercept, $\alpha$ (i.e., the mean value of $d$ ), the significance of which was assessed via t-test. To the extent that the nuisance covariates can explain between-subject variance in $d$, inclusion of the $Z \gamma$ term reduces $\varepsilon$ and increases the t-score for $\alpha$ (at the expense of lower degrees of freedom). To control for multiple comparisons, a nonparametric approach was employed based on the wild boostrap (Eklund et al., 2016) and threshold-free cluster enhancement (TFCE; Smith and Nichols, 2009). At each cortical surface node, a null distribution of t-scores was obtained from

$$
S d=\alpha+Z \gamma+\varepsilon
$$


where $S$ is a sign-flip matrix drawn from 10000 randomly generated sign-flip permutations. The t-score maps obtained from Eqs. 3-4 were converted to z-scores and processed with TFCE using the CoSMoMVPA toolbox v1.1.0 (Oosterhof et al., 2016) within MATLAB R2018a, resulting in one true and 10000 null TFCE maps. A single null TFCE distribution was formed by obtaining the brain-wide maximum TFCE value from each null TFCE map. Finally, a TFCEcorrected z-score map was generated from the proportion of null TFCE values exceeding the true TFCE value at each surface node. In fact, TFCE was applied separately at positive- and negative-valued surface nodes in the original t-score map, yielding a single TFCE z-score map whose two-tailed $p$-values were corrected to control the familywise error rate (FWER). The corrected TFCE z-score map was thresholded at $|z|>1.96$ (FWER-corrected $p<0.05$ ).

\subsubsection{Unsupervised Clustering of STRFs}

Once cortical surface nodes showing a significant difference in $R^{2}$ between Competing and Unison were identified, the STRFs at these nodes were obtained as in 2.3.4.3. At the participant level, these STRFs were normalized to have unit vector length across the 120 STRF features. The normalized STRFs were then averaged across subjects separately for the Competing and Unison conditions. It was presumed that differences in $R^{2}$ between Competing and Unison might also be reflected in STRF differences between Competing and Unison. Thus, 'mean-difference STRFs' were obtained by subtracting the Unison STRFs from the Competing STRFs. It was further presumed that different patterns of STRF differences might be observed across cortical surface nodes. Thus, the mean-difference STRFs were submitted to an unsupervised agglomerative clustering analysis. Clustering was performed separately for the surface nodes at which Competing > Unison and Unison > Competing relative to $R^{2}$. The cluster model was implemented with the evalclusters.m function in the Matlab Statistics and Machine Learning Toolbox. Clusters were defined based on the Euclidean distance with a Ward linkage. For a maximum of 10 clusters, the optimal solution was taken to be that number of clusters that maximized the Silhouette index (Rousseeuw, 1987) averaged across all observations.

\subsubsection{Predicting Behavior from fMRI Data: Hierarchical Bayesian Regression}

Clustering analysis identified three brain networks that contribute differentially to recognition of speech with simultaneous talkers. We refer to these as the 'Intelligibility network,' 'Distortion network,' and 'Semantic network,' respectively. To determine whether the BOLD signal within these networks could be used to predict trial-by-trial behavior (correct/incorrect button presses) in the CRM task, a hierarchical Bayesian regression approach was employed. To this end, a beta time series was estimated for each participant using the least squares-separate (LSS; Mumford et al., 2012) approach as implemented in AFNI with a duration-modulated BLOCK hemodynamic response function and stimulus onsets and durations estimated as described in 2.3.4.1. The output beta time series at each surface node represents the overall magnitude of the BOLD response to each experimental trial over the duration of the experiment. A second, simulated beta time series was estimated for each participant using that participant's STRFs estimated as in 2.3.4.3. At each surface node, the beta weight for a given trial, $i$, was obtained as the dot product of the vectorized STRF and the vectorized bubbles filter presented on that trial, Bub: 


$$
\beta_{i}=S T R F \cdot B u b_{i}
$$

The STRF-simulated beta time series can be interpreted in the same way as the LSS time series but reflects only those trial-by-trial fluctuations in BOLD activity that are driven by the acoustic distortions introduced by bubbles; the LSS coefficients, on the other hand, reflect any information processing that might modulate the BOLD signal from trial to trial.

These beta time series (LSS, STRF) were obtained separately at each cortical surface node; univariate beta time series for each cortical network (Intelligibility, Distortion, Semantic) were obtained for each participant by averaging the beta coefficients across the nodes in those networks at each time point (trial). These univariate time series were entered as predictors in a presses scored as correct or incorrect. Unaggregated data from all trials $(N=400)$, both conditions (Unison, Competing), and all participants $(\mathrm{N}=25)$ were entered in a single regression model (20000 total observations):

$$
r=0.33+0.67 *\left(\frac{1}{1+\exp (-\eta)}\right)
$$

where binary responses, $r$, are predicted with a logistic link function that can take values in the range 0.33 (the guessing rate in a 3-AFC task) to 1 (ceiling performance). The responses were taken to be Bernoulli distributed. The linear term, $\eta$, was modeled hierarchically with both fixed (group-level) and random (participant-level) effects.

Two classes of model were estimated that we term 'network-within, signal-between' and 'network-between, signal-within.' The 'network-within, signal-between' models were defined as follows:

$$
\begin{gathered}
\eta \sim 0+\text { Intel } * \text { Condition }+ \text { Distort } * \text { Condition }+ \text { Seman } * \text { Condition } \\
+(0+\text { Intel } * \text { Condition }+ \text { Distort } * \text { Condition }+ \text { Seman } * \text { Condition } \mid \text { participant })
\end{gathered}
$$

Where 0 represents a suppression of the intercept term, Condition is an effect-coded categorical variable (Unison $=-1$, Competing $=1$ ), Intel is the beta time series from the Intelligibility network, Distort is the beta time series from the Distortion network, Seman is the beta time series from the Semantic network, “*' represents an expanded interaction (main effects plus two-way interaction), and (... | participant) denotes the random effect terms (i.e., all effects in the model were estimated for the group and for each participant as deviations from the group terms). Two versions of the model in Eq. 6 were estimated: one where Intel, Distort, and Seman were the LSS beta time series, and one where they were the STRF beta time series. The 'networkbetween, signal-within' models were defined as follows: 


$$
\begin{array}{r}
\eta \sim 0+L S S * \text { Condition }+S T R F * \text { Condition } \\
+(0+L S S * \text { Condition }+S T R F * \text { Condition } \mid \text { participant })
\end{array}
$$

where LSS is the LSS-derived beta time series from one of the three brain networks, STRF is the STRF-derived beta time series from one of the three brain networks, and the remaining terms and symbols are defined as in Eq. 7. Three versions of the model in Eq. 8 were estimated: one where LSS and STRF were drawn from the Intelligibility network, one where they were drawn from the Distortion network, and one where they were drawn from the Semantic network. Finally, individual models subsumed by the 'network-within, signal-between' and 'network-between, signal-within' models were estimated as:

$$
\eta \sim 0+\text { Beta } * \text { Condition }+(0+\text { Beta } * \text { Condition } \mid \text { participant })
$$

where Beta was one of six possible beta time series (Intelligibility-LSS, Intelligibility-STRF, Distortion-LSS, etc.) and the remaining terms and symbols were defined as in Eqs. 7 and 8. A null model was also estimated including only the Condition terms. For all models other than the null, the beta time series predictors were normalized (z-score) within condition and participant prior to model fitting.

All models were fit in a Bayesian context using No-U-Turn sampling as implemented in Stan (Hoffman and Gelman, 2014) to estimate the posterior distribution of model parameters. Calls to Stan were generated automatically via the brms package v2.13.5 (Bürkner, 2017) in $R$ v3.6.1 (R Core Team, 2019), which uses a model formula syntax like that used in Eqs. 7-9. Weakly informative Gaussian priors, $N(0,5)$, were set on all fixed effects parameters and the brms default priors were set otherwise. For all models, 10 independent sampling chains were obtained, each with 1000 warmup and 2000 post-warmup samples, resulting in 20000 total draws from the posterior. Model convergence was assumed when posterior chains were visually well mixed, Gelman-Rubin $\hat{R}$ less than 1.01 for all parameters, adequate (>1000) bulk and tail effective sample size was achieved for all parameters, and few $(<5)$ or no divergent transitions were observed. Leave-one-out cross-validation was performed using Pareto smoothed importance sampling via the loo package v2.3.1 (Vehtari et al., 2017) in $R$. Model fit was evaluated using the leave-one-out expected log pointwise predictive density (elpd_loo) and model averaging was performed via stacking (Yao et al., 2018) to determine the weighted combinations of models that maximized the elpd_loo. A posterior predictive check was performed by obtaining the median of the expected posterior predictive distribution and calculating the area under the receiver operating characteristic curve (AUC) over all observations.

\section{RESULTS}

\subsection{Audiograms and Behavioral Results}

The sample mean air conduction audiogram (better ear) is shown in Figure 3A along with the sample standard deviation and range at each audiometric frequency. As a group, these listeners had normal hearing up to $1 \mathrm{kHz}$ with a mild sloping loss at higher frequencies. As indicated by the range, some listeners had moderate losses at frequencies $2 \mathrm{kHz}$ and above, but most often 
at 6 and $8 \mathrm{kHz}$; in addition, some listeners had normal thresholds across all audiometric frequencies. All listeners' thresholds met the inclusion criteria outlined in 2.1.2. In general, these listeners can be considered to have normal or near-normal hearing.

\section{1}

792

793

794

795

796

797

798

799

800

801

802

803

804

805

806

807

808

809

810

811

812

813

814

815

816

817

818

819

820
A

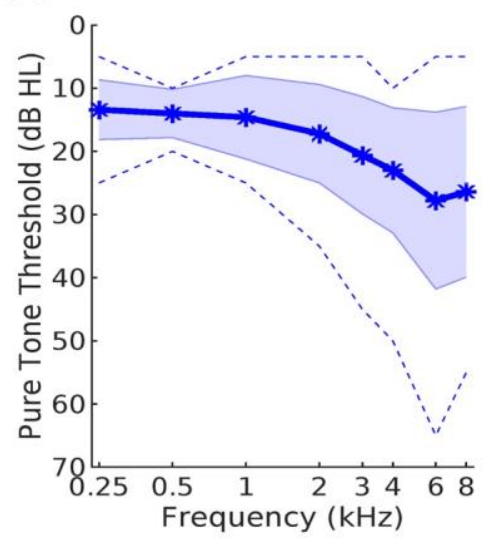

B

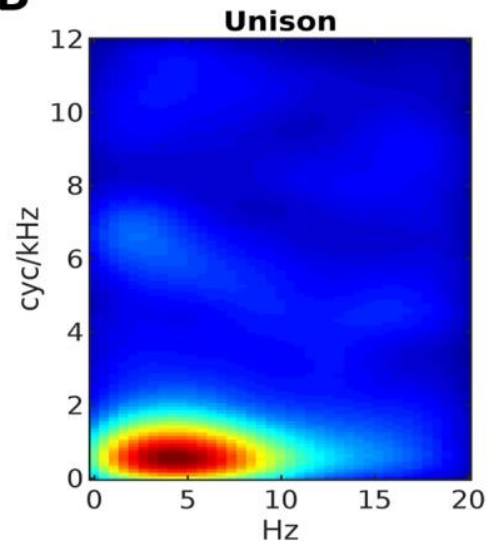

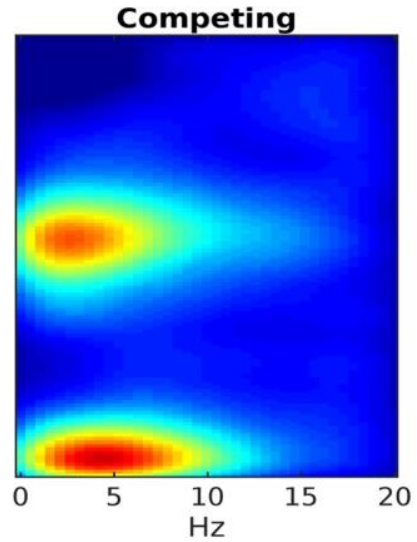

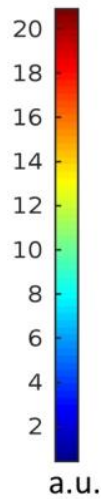

Figure 3. (A) Mean better-ear air-conduction audiogram (bold blue) across all participants. The blue shaded region reflects $+/-1 S D$ and the dashed lines show the range across all participants. (B) Across-participant mean behavioral classification images in the Unison (left panel) and Competing (right panel) conditions. The heatmap reflects the extent to which a given MPS region (cf., Figure 1) contributes to behavioral performance on the CRM task, with warm colors reflecting a strong positive association with speech recognition performance.

The mean percent-correct performance in the Competing condition was $66.8 \%$ ( $\mathrm{sd}=1.3 \%$, range $=64.0-68.3 \%$ ), indicating convergence of the weighted staircase procedure for all participants. The mean percent-correct performance in the Unison condition was $84.3 \%$ (sd = $4.4 \%$, range $=77.8-92.8 \%$ ). As expected, performance in the Unison condition was uniformly better than in the Competing condition. The magnitude of this difference was somewhat variable across participants ( 10-25\%), but this was also expected given that performance was not constrained in the Unison condition. The threshold number of bubbles in the Competing condition was quite variable across participants (mean $=97.4$, $\mathrm{sd}=31.4$, range $=60.0-171.3$ ). For reference, this corresponds to considerable distortion tolerated at the bottom end of the range ( 60 bubbles) and very little distortion tolerated at the top end of the range ( $>150$ bubbles), with an intermediate degree of distortion tolerated ( 100 bubbles) on average. Such a range is expected in a heterogenous group of listeners (Venezia et al., 2019a; Venezia and Leek, 2020), especially given the additional background noise produced by the scanner. Consistent with our previous studies using a single talker in quiet, the mean behavioral classification image for the Unison condition (Figure 3B, left) revealed strong contributions to performance from only the 'phonetic-content' region of the MPS, with weights centered on $\sim 4 \mathrm{~Hz}$ in the temporal modulation dimension and $<2 \mathrm{cyc} / \mathrm{kHz}$ in the spectral modulation dimension (Venezia et al., 2016, 2019a). This same region was observed in the mean classification image for the Competing condition (Figure 3B, right), but additional strong contributions to performance from the target (female) talker region of the MPS were also revealed at $\sim 6.5 \mathrm{cyc} / \mathrm{kHz}$ and $<10 \mathrm{~Hz}$ (cf., Venezia et al., 2020). This result confirms strong task-related differences in acousticspeech information processing between the Unison and Competing conditions. That is, the conditions differ beyond overall task performance or difficulty. 


\subsection{Spectrotemporal Receptive Field Modeling}

\subsubsection{Model Predictions}

The group mean $R^{2}$ maps for the Unison and Competing conditions are shown in Figure $4 \mathrm{~A}$ and Figure 4B, respectively. These maps show regions for which STRF models were best able to predict the brain response from trial-by-trial acoustic distortion patterns imposed by bubbles. In both conditions, STRF predictive power was highest bilaterally in peri-Sylvian auditory and speech regions including Heschl's gyrus and surrounds, superior temporal gyrus (STG), and superior temporal sulcus (STS). The STG effects were more pronounced in the Competing condition. In the Unison condition, additional non-trivial values were observed in the bilateral insula, frontal operculum, middle frontal gyrus, precentral gyrus, supplementary motor area, inferior parietal lobe, and anterior and posterior midline regions. Indeed, when $R^{2}$ was contrasted directly between the Unison and Competing conditions (Figure 4C), significantly larger values were observed for Competing than Unison (yellow) in the bilateral STG spanning from mid-anterior to posterior subregions, and significantly larger values were observed for Unison than Competing (blue) in the bilateral insula, frontal operculum, inferior frontal sulcus, middle frontal gyrus, precentral gyrus, supplementary motor area, ventromedial prefrontal cortex, inferior parietal lobe, and left posterior cingulate cortex. It was somewhat paradoxical to observe higher values in frontoparietal and midline regions given that such regions are generally modulated by task difficulty, but here task difficulty was greater in the Competing condition than the Unison condition. This will be addressed in the next subsection. 


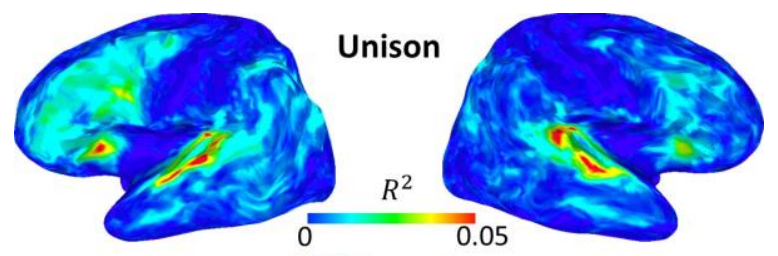

A
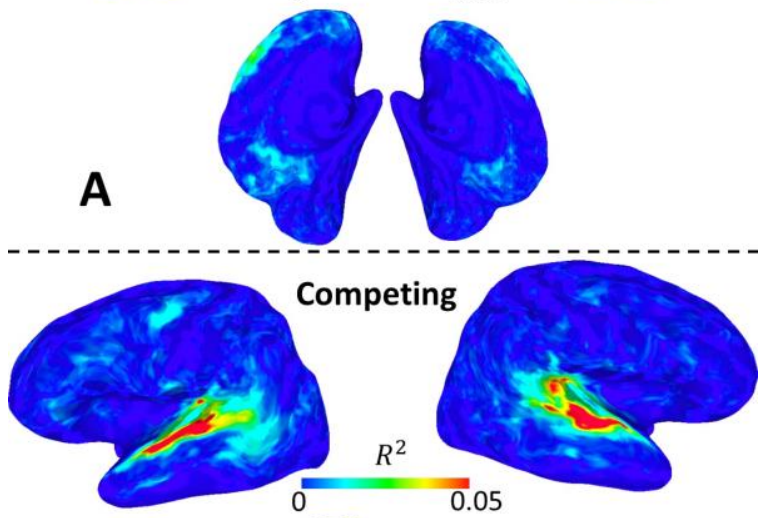

845

C

B
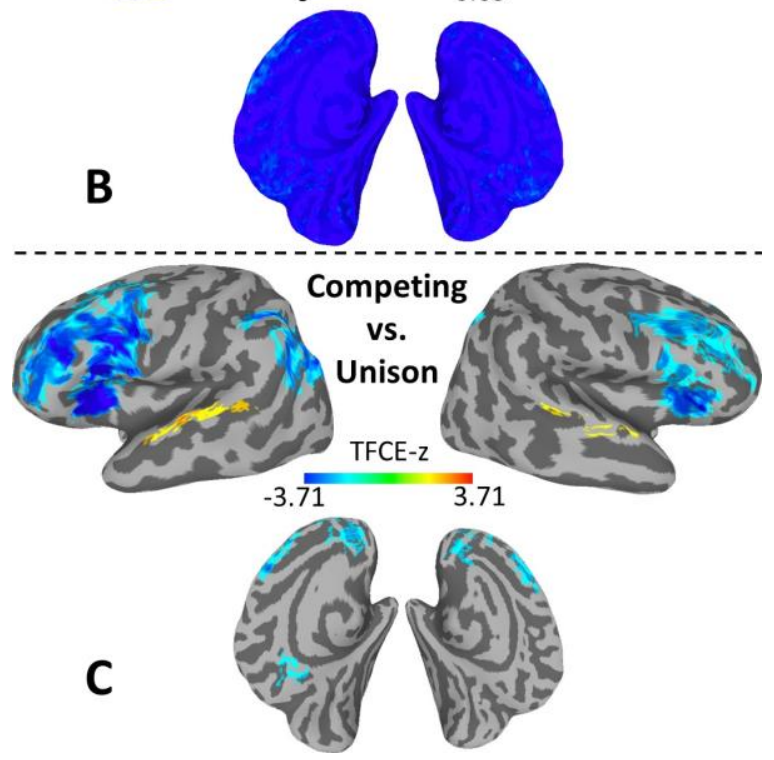

846

847

848

849

850

851

Figure 4. (A) Second-level maps of the mean STRF model prediction accuracy in the Unison condition $\left(R^{2}\right.$, uncorrected). (B) Second-level maps of the mean STRF model prediction accuracy in the Competing condition ( $R^{2}$, uncorrected). (C) Second-level contrast of STRF model prediction accuracy $\left(R^{2}\right)$ in the Competing condition vs. the Unison condition, displayed as TFCE-corrected $z$-scores (FWER-corrected $p<0.05$ ). All plots displayed on an inflated cortical surface rendering of the Colin 27 template in MNI space.

852

853

854

855

856

857

858

859

860

3.2.2. Spectrotemporal Receptive Fields

To determine whether and how the differences between Unison and Competing were driven by differences in STRF patterns across participants, agglomerative clustering was performed on Competing minus Unison 'difference STRFs' (2.3.6). Clustering was performed separately on cortical surface nodes implicated in the Competing > Unison and Unison > Competing $\mathrm{R}^{2}$ contrasts. It is important to note that such clustering does not enforce any spatial or brainanatomical constraints on the solution and is based entirely on STRF similarity (i.e., functional 
response properties) at the level of individual cortical surface nodes. In fact, no stable clustering solution was identified for nodes from the Competing $>$ Unison $\mathrm{R}^{2}$ contrast. All mean Silhouette indices were less than 0.3 and the individual clusters were non-uniform with respect to the Silhouette index with many individual nodes falling far below the mean. As such, all nodes from the Competing > Unison $\mathrm{R}^{2}$ contrast were treated as a single STG network (Figure 5A, red). For nodes from the Unison $>$ Competing $\mathrm{R}^{2}$ contrast, the optimal solution contained two clusters (mean Silhouette index $=0.51$ ). When plotted on the cortical surface, these clusters clearly fell into two distinct networks: a frontoparietal 'sensorimotor speech' network including the insula, frontal operculum, precentral gyrus, middle frontal gyrus, supplementary motor area, and intraparietal sulcus (Figure 5A, blue); and a 'semantic memory' network in the left angular and supramarginal gyri, bilateral ventromedial prefrontal cortex, left posterior cingulate cortex, and left superior frontal gyrus (Figure 5A, green; Binder and Desai, 2011).
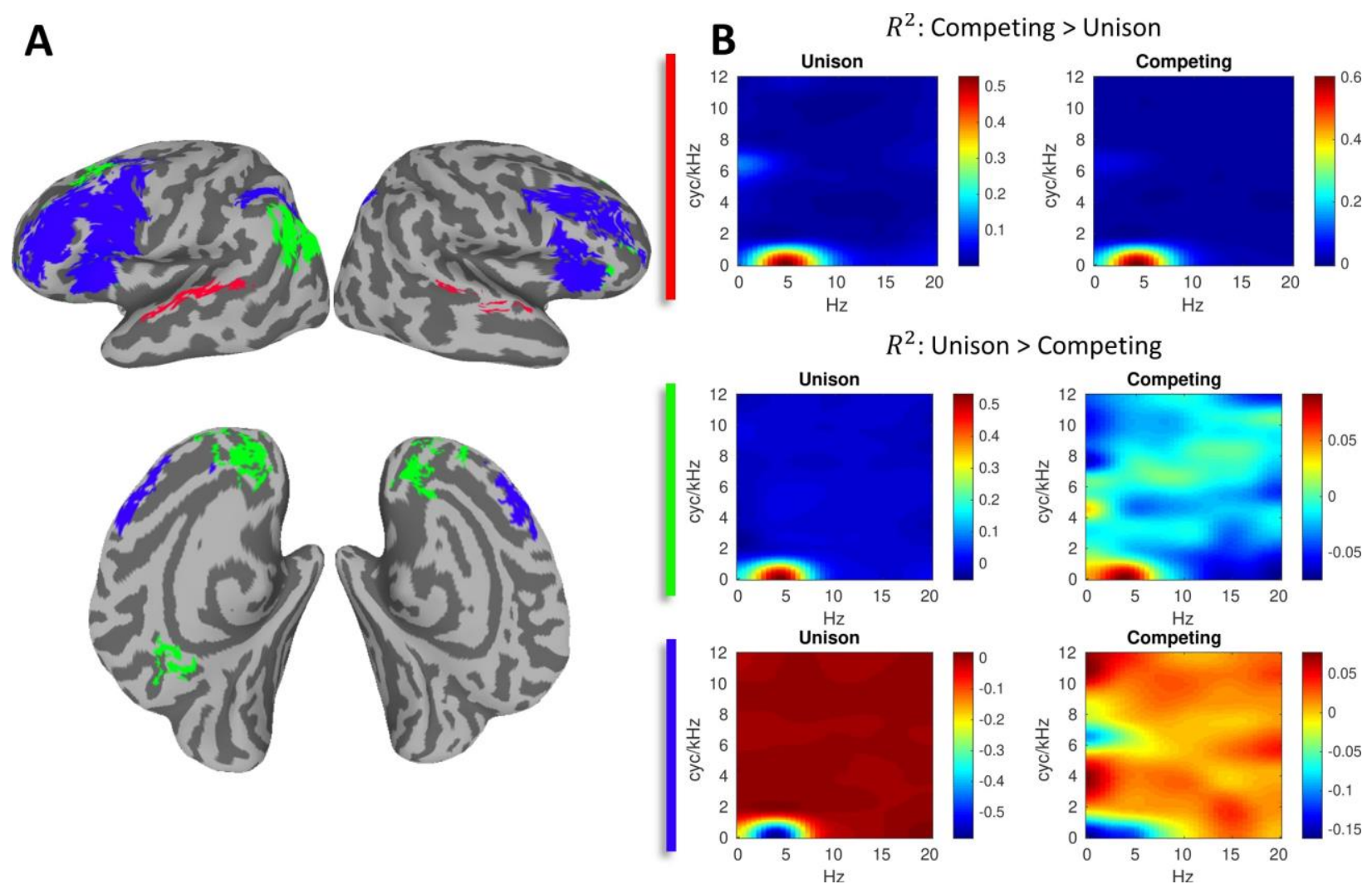

Figure 5. (A) Results of agglomerative clustering analysis applied to second-level 'difference STRFs' (Competing minus Unison), which identified three distinct brain networks: Intelligibility (red), Distortion (blue), and Semantic (green). Plots are displayed on an inflated cortical surface rendering of the Colin 27 template in MNI space. (B) Network-mean STRFs (i.e., averaged across nodes in the network and participants) in the Unison (left column) and Competing (right column) conditions, shown separately for the Intelligibility (red bar), Semantic (green bar) and Distortion (blue bar) networks. Networks are separated by the sign of the difference in STRF model prediction accuracy ( $R^{2}$; cf., Figure $4 C$ ) between the Competing and Unison conditions (Competing > Unison: Intelligibility network; Unison > Competing: Semantic/Distortion networks). 
For each of the three cortical networks plotted in Figure 5A, the group average STRFs are plotted in Figure 5B separately for Unison and Competing after normalizing to unit vector length at each individual surface node to emphasize STRF patterns rather than STRF magnitude. In the STG network (Figure 5B, top row), nearly identical STRF patterns were observed in the Unison and Competing conditions. Specifically, STRF weight was assigned primarily to the 'phonetic content' region of the MPS centered at $\sim 4 \mathrm{~Hz}$ and $<2 \mathrm{cyc} / \mathrm{kHz}$, just as in the behavioral classification images. Indeed, our previous work shows this MPS region is critical to speech intelligibility for a single talker in quiet (Venezia et al., 2016, 2019a). This explains why clustering of STG nodes failed - these nodes respond rather uniformly to intelligible speech across subregions of the STG and across experimental conditions. It is likely that differences in STRF amplitude, rather than pattern, explain the differences between conditions. Thus, we henceforth refer to this STG network as the 'Intelligibility' network.

In the semantic memory network (Figure 5B, middle row), STRF patterns were similar to those in the Intelligibility network, particularly in the Unison condition. The STRF in the Competing condition was noisier and contained (marginal) additional weight on the target (female) talker region of the MPS, similar to the pattern in the behavioral classification image for Competing (cf., Figure 3B). This suggests the semantic memory network responds to intelligible speech in a task dependent manner. Henceforth, we refer to this network as the 'Semantic' network.

Finally, in the frontoparietal network (Figure 5B, bottom row), STRF patterns were noticeably different than in the other networks and noticeably different between conditions. In the Unison condition, negative weight was assigned to the phonetic speech region of the MPS revealing essentially an opponent STRF compared to the Intelligibility- and Semantic-network STRFs, suggesting this network responds less to intelligible speech and more to distorted speech. In the Competing condition, negative STRF weights were assigned to the phonetic-content and targettalker MPS regions, and positive STRF weights were assigned to the competing-talker (male) MPS region, suggesting again that the frontoparietal network responds to acoustic distortion but in a task dependent manner - i.e., in the Competing condition, distortion can be generated by removing the phonetic content, removing the target talker's voice, or retaining the competing talker's voice. This potentially explains why $R^{2}$ values were larger for Unison than Competing in this network, since the primary source of distortion in the Unison condition was the acoustic distortion imposed by bubbles, while in the Competing condition distortion could be caused by bubbles and by additional sources such as informational masking (i.e., interference from the Competing talker at higher, 'post-acoustic-phonetic' levels of speech processing or within regions of the MPS such as the phonetic content region where the target and competing signals cannot be separated). Henceforth, we refer to the frontoparietal network as the 'Distortion' network.

\subsection{Predicting Behavior from fMRI Data}

In this section, outcomes of hierarchical Bayesian logistic regression modeling are presented wherein trial-by-trial 'beta time series' extracted from the Intelligibility, Distortion, and Semantic networks were used to predict trial-by-trial performance across both conditions and all participants. The beta time series were estimated either via LSS, in which case the time series 
reflected BOLD activation from any source time-locked to the stimuli, or via simulation from STRFs, in which case the time series reflected the BOLD activation predicted just from the acoustic distortion introduced by bubbles. The conceptual motivation for fitting separate LSS and STRF models was to distinguish between contributions to behavior from brain activity that is predictable from acoustic distortion patterns alone (STRF) versus brain activity that is not predictable from acoustic distortion patterns alone (LSS).

\subsubsection{Network-Within, Signal-Between}

Outcomes for the 'network-within, signal-between' models are presented in Figure 6 (posterior marginal effects) and Table 1 (fixed effects regression coefficients and model fit metrics). In these models, beta time series from all three brain networks - Intelligibility, Distortion, and Semantic - were entered in the same model (Eq. 7) and separate models were estimated for the two types of time series (LSS, STRF). Thus, the three brain networks competed with one another to explain trial-by-trial differences in behavior; differential contributions of the three brain networks to performance can be compared within a given model by examining the regression coefficients. Differential contributions of the two signal types (LSS, STRF) to behavior can be assessed via model comparison (elpd_loo, stacking weights).

Figure 6 can be interpreted as a series of 'neurometric' functions that characterize how task performance changes as a function of the relative trial-by-trial activation in each brain network. The different models within the class 'network-between, signal-within' (LSS/STRF) are labeled with uppercase letters (A/B). In each panel, fixed (group mean) effects are plotted as bold lines (posterior median; shaded regions $=95 \%$ credible interval) and random (individual-participant) effects are plotted as dashed lines (posterior median). In Table 1, only the fixed effects regression coefficients are presented. Roughly, a coefficient can be interpreted as 'significant' in the frequentist sense if the $95 \%$ credible interval - given parenthetically in Table 1 - does not contain zero. Note, the null model is presented in Table 1 but not shown in Figure 6 because it only contains predictors representing overall performance in each condition (Condition-U, Condition-C), but does not contain predictors representing brain-network activation. The Condition- $U$ and Condition- $C$ coefficients are presented in units of proportion-correct performance - they describe the group-mean proportion correct when each of the three brain networks takes its mean activation amplitude across trials; in other words, these coefficients indicate the height of the neurometric functions at their center (zero on the abscissa), separately for Unison (Condition- $U$ ) and Competing (Condition-C). The Intel, Distort, and Seman coefficients are presented in log-odds units and are proportional to the overall slopes (ignoring experimental condition) of the neurometric functions in the Intelligibility, Distortion, and Semantic brain networks, respectively. Strictly, these coefficients indicate the change in performance (as log-odds) given a one-standard-deviation increase in across-trial activation within a given brain network. The Condition:Intel, Condition:Distort, and Condition:Seman coefficients indicate how the slopes of the neurometric functions change by condition in each of the three brain networks. These coefficients should be added to the overall slope coefficients to obtain the Unison slope and subtracted from the overall slope coefficients to get the Competing slope. A positive (negative) slope indicates that performance increases (decreases) with increasing activation 
974 within a given brain network and experimental condition. A larger absolute value of the slope indicates a stronger relationship between activation and performance.

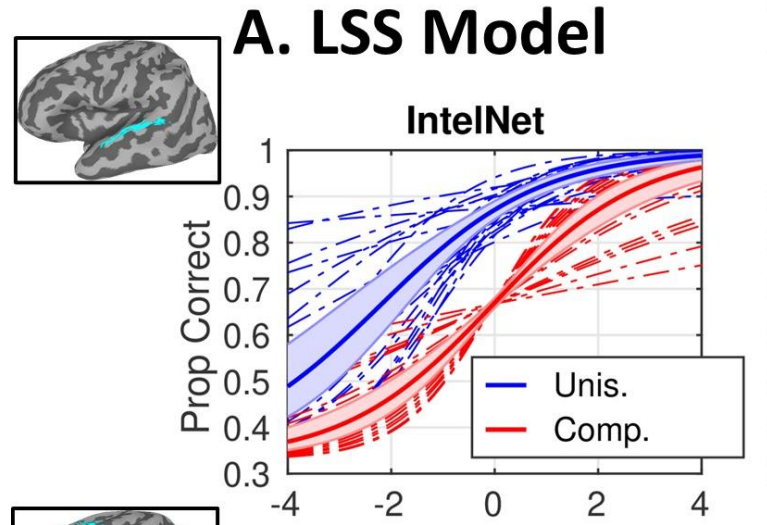

\section{B. STRF Model}
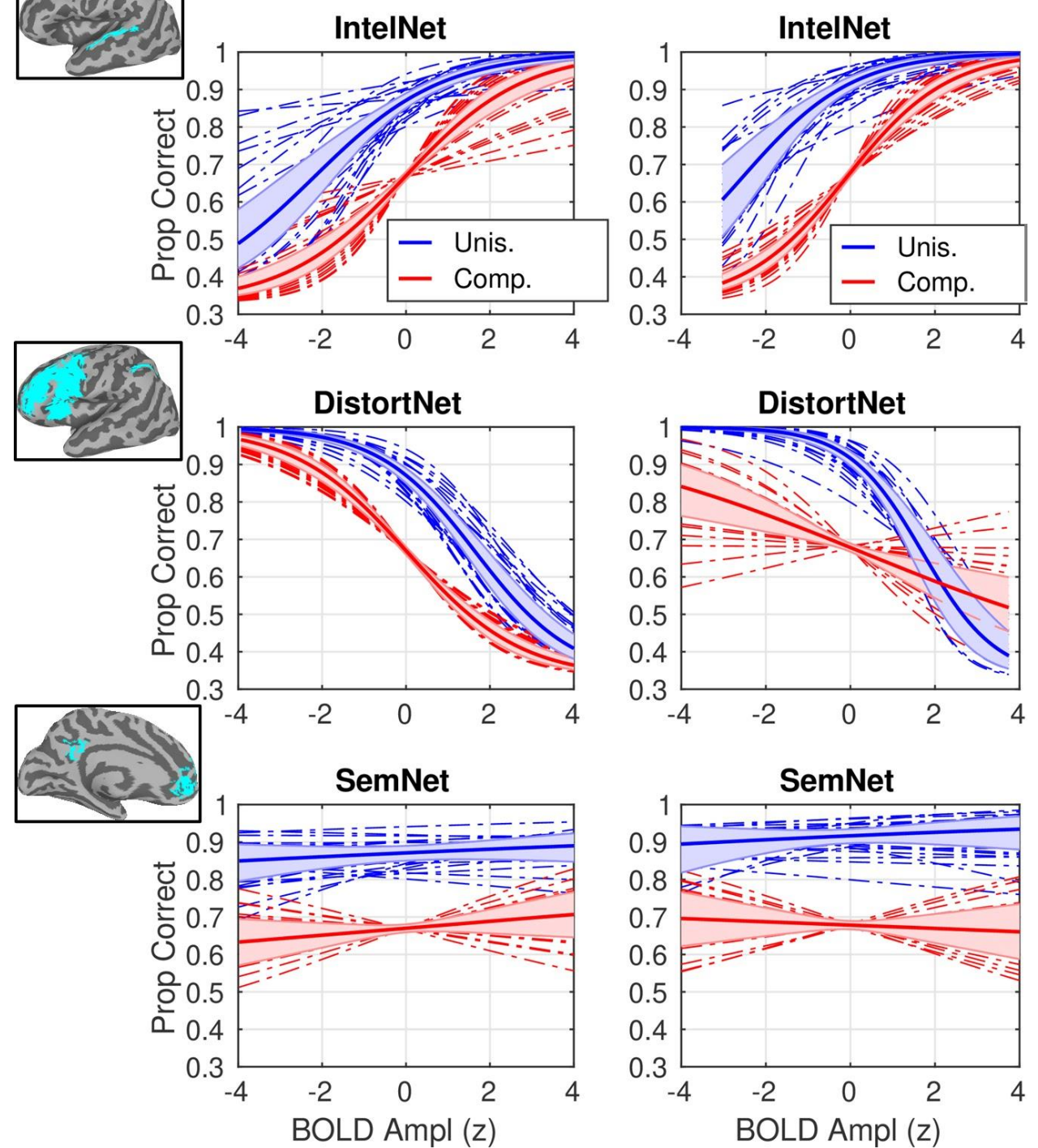

\section{SemNet}

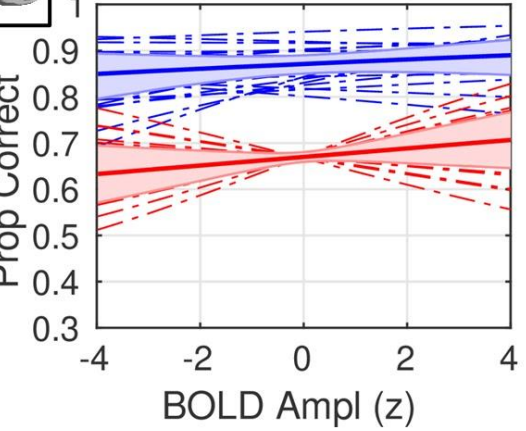

Figure 6. Results of Bayesian hierarchical logistic regression models ('network-within, signal-between') showing the relation between trial-by-trial network activation and behavioral performance (proportion correct in the 3-AFC CRM task). Each panel plots z-scored BOLD activation amplitude (abscissa) against proportion correct performance (ordinate) in the Unison (blue) and Competing (red) conditions. Bold lines (posterior median) and shaded regions (95\% posterior credible interval) reflect the group-level (fixed) effect, and dashed lines reflect the subject-level (random) effects (posterior median). (A) Results of the LSS model, which estimates trial-level activation using a standard event-related analysis (i.e., activation to any source time-locked to the stimulus). (B) Results of the STRF model, which simulates trial-level activation from the dot product of the network STRF and the bubbles filter pattern presented on each trial. For $A / B$, the brain networks are separated by row as shown in the insets. IntelNet = Intelligibility network, DistortNet = distortion network, SemNet = Semantic network. 
As is clear from Figure 6 and the regression coefficients in Table 1, activation in the Intelligibility and Distortion networks has a strong relation with performance, but in opposite directions performance increases with increasing activation in the Intelligibility network and decreases with increasing activation in the Distortion network. For the Intelligibility network, this pattern is essentially unchanged whether we consider the LSS model or the STRF model. If anything, the relation between activation in the Intelligibility network and performance is stronger in the STRF model. For the Distortion network, the relation between activation and performance differs in the STRF model compared to the LSS model: in the Unison condition, the relation is stronger, but in the Competing condition the relation is much weaker (see Condition: Distort for the STRF model in Table 1). A more rigorous comparison of the LSS and STRF coefficients within each network can be gleaned from the 'network-between, signal-within' models presented in the next subsection. Finally, for the Semantic network, there was no significant relation between activation and performance.

Table 1. Regression coefficients and model fit metrics for the 'network-within, signal-between' model class.

\begin{tabular}{|c|c|c|c|c|}
\hline & \multicolumn{4}{|c|}{ Model Name } \\
\hline & LSS & STRF & Null & Stacked \\
\hline \multicolumn{5}{|l|}{ Coefficient } \\
\hline $\begin{array}{l}\text { Condition-U } \\
(95 \% \mathrm{CI})\end{array}$ & $\begin{array}{c}0.87 \\
(0.85,0.89)\end{array}$ & $\begin{array}{c}0.92 \\
(0.90,0.94)\end{array}$ & $\begin{array}{c}0.85 \\
(0.83,0.87)\end{array}$ & -- \\
\hline $\begin{array}{l}\text { Condition-C } \\
(95 \% \mathrm{CI})\end{array}$ & $\begin{array}{c}0.67 \\
(0.66,0.68)\end{array}$ & $\begin{array}{c}0.68 \\
(0.67,0.69)\end{array}$ & $\begin{array}{c}0.67 \\
(0.66,0.68)\end{array}$ & -- \\
\hline $\begin{array}{l}\text { Intel } \\
(95 \% \mathrm{CI})\end{array}$ & $\begin{array}{c}0.68 \\
(0.54,0.83)\end{array}$ & $\begin{array}{c}0.80 \\
(0.66,0.95)\end{array}$ & -- & -- \\
\hline $\begin{array}{l}\text { Distort } \\
(95 \% \mathrm{CI})\end{array}$ & $\begin{array}{c}-0.80 \\
(-0.89,-0.71)\end{array}$ & $\begin{array}{c}-0.71 \\
(-0.85,-0.58)\end{array}$ & -- & -- \\
\hline $\begin{array}{l}\text { Seman } \\
(95 \% \mathrm{CI})\end{array}$ & $\begin{array}{c}0.05 \\
(-0.01,0.12)\end{array}$ & $\begin{array}{c}0.02 \\
(-0.08,0.13)\end{array}$ & -- & -- \\
\hline $\begin{array}{l}\text { Condition:Intel } \\
(95 \% \mathrm{CI})\end{array}$ & $\begin{array}{c}-0.03 \\
(-0.09,0.04)\end{array}$ & $\begin{array}{c}-0.04 \\
(-0.13,0.07)\end{array}$ & -- & -- \\
\hline $\begin{array}{l}\text { Condition:Distort } \\
(95 \% \mathrm{CI})\end{array}$ & $\begin{array}{c}-0.06 \\
(-0.12,0.00)\end{array}$ & $\begin{array}{c}-0.43 \\
(-0.59,-0.28)\end{array}$ & -- & -- \\
\hline $\begin{array}{l}\text { Condition:Seman } \\
(95 \% \mathrm{CI})\end{array}$ & $\begin{array}{c}-0.00 \\
(-0.07,0.06)\end{array}$ & $\begin{array}{c}0.05 \\
(-0.04,0.14)\end{array}$ & -- & -- \\
\hline \multicolumn{5}{|l|}{ Model Fit } \\
\hline elpd_loo diff (SE) & $-450.9(51.2)$ & 0 & $\begin{array}{c}-1175.9 \\
(50.0)\end{array}$ & -- \\
\hline Stacking $w$ & 0.25 & 0.75 & 0 & -- \\
\hline AUC & 0.73 & 0.78 & 0.64 & 0.78 \\
\hline
\end{tabular}

In Table 1, three 'Model Fit' metrics are presented that directly compare the LSS, STRF, and null models. The elpd_loo is a fully cross-validated measure of model predictive performance (larger = better performance). It is presented in terms of the pairwise difference between each 
model and the best performing model, which is set to zero. In general, if the pairwise difference is more than twice the standard error of the difference (given parenthetically in Table 1), the best model performs significantly better than its comparator. The stacking weights sum to one and indicate the weighted combination of models that best predicts unseen data (i.e., maximizes elpd_loo). If weight is assigned to more than one model, it indicates that the combination of models is better able to predict (held-out) task performance than any single model (although equal weights can mean different models make equally good predictions). The AUC is a posterior predictive check showing the unbiased classification accuracy (larger = better) of the model considering only the data used to train the model (i.e., without cross-validation). As is clear from Table 1, the STRF model is clearly the best performing model. It has a significantly larger elpd_loo than the LSS and null models, the largest stacking weight at 0.75 , and the best AUC. However, the LSS model also outperforms the null model (elpd_loo and AUC) and has a non-trivial stacking weight of 0.25 . This suggests that the LSS and STRF signals account for partially independent components of task performance. In general, the STRF model seems to benefit from better predictions in the Intelligibility network (overall) and the Distortion network (Unison condition), while the LSS model benefits from better predictions in the Distortion network (Competing condition). Interestingly, the stacked model does not outperform the STRF model in terms of the AUC, but this does not trivialize the stacking weights given that the AUC metric is not cross-validated.

\subsubsection{Network-Between, Signal-Within}

Outcomes for the 'network-between, signal-within' models are presented in Figure 7 and Table 2, which can be interpreted just as Figure 6 and Table 1. In these models, beta time series representing the two signal types (LSS, STRF) were entered in the same model (Eq. 8) and separate models were estimated for each brain network (Intelligibility, Distortion, and Semantic). Thus, the two signal types competed with one another to explain trial-by-trial differences in behavior within a given brain network; differential contributions of the two signal types can be compared within a given model by examining the regression coefficients. Differential contributions of the different brain networks (Intelligibility, Distortion, and Semantic) to behavior can be assessed via model comparison (elpd_loo, stacking weights). 


\section{A. IntelNet Model}

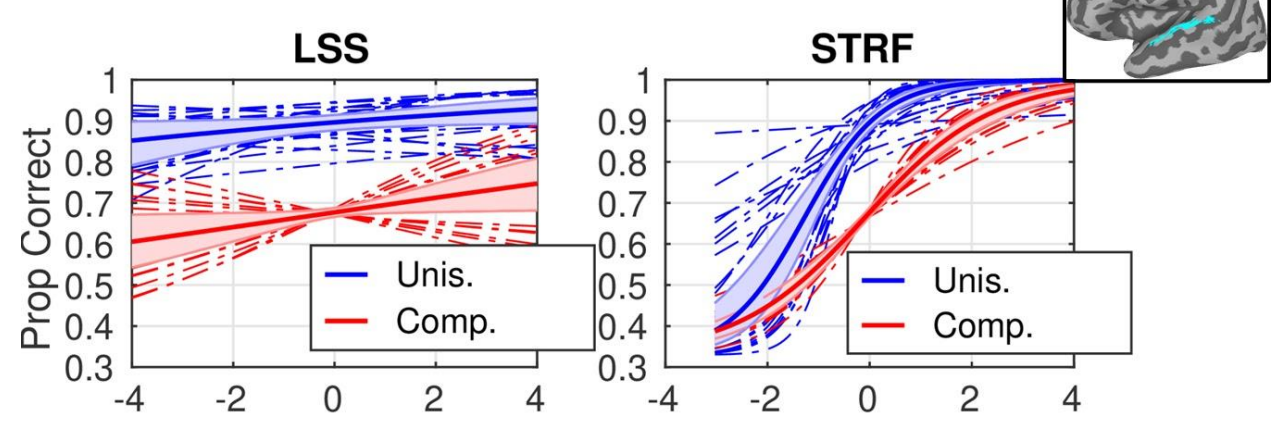

\section{B. DistortNet Model}

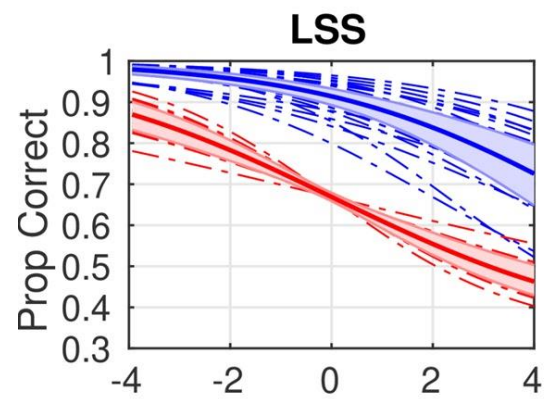

\section{SemNet Model}
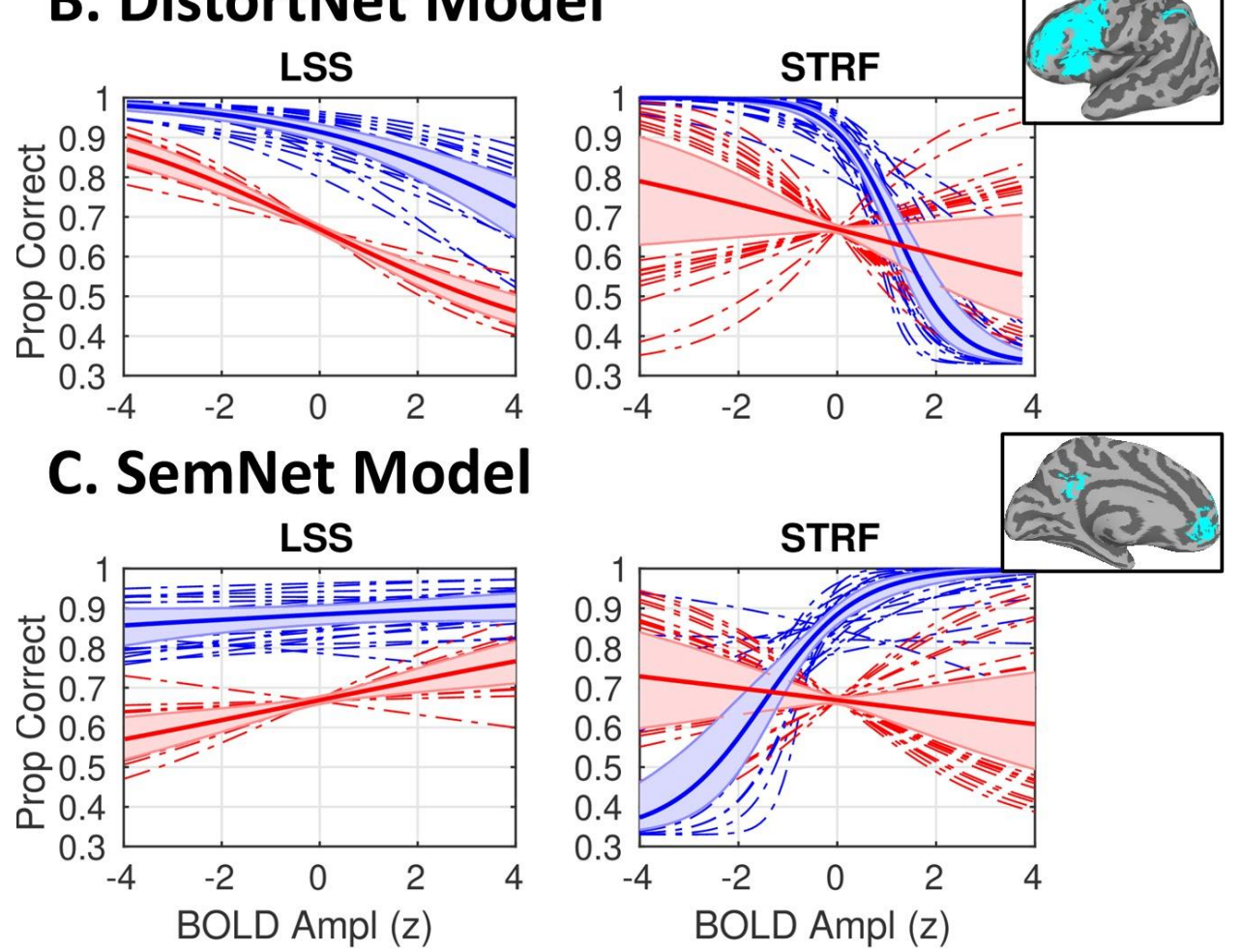

1043

1044

1045

1046

1047

1048

1049

1050

1051

1052

1053

1054

1055

1056

Figure 7. Results of Bayesian hierarchical logistic regression models ('network-between, signal-within') showing the relation between trial-by-trial network activation and behavioral performance (proportion correct in the 3-AFC CRM task). Each panel plots z-scored BOLD activation amplitude (abscissa) against proportion correct performance (ordinate) in the Unison (blue) and Competing (red) conditions. Bold lines (posterior median) and shaded regions (95\% posterior credible interval) reflect the group-level (fixed) effect, and dashed lines reflect the subject-level (random) effects (posterior median). (A) Results of the IntelNet model, which compares the effects of the LSS-and STRF-estimated activation time series within the Intelligibility network (inset). (B) Results of the DistortNet model, which compares the effects of the LSS- and STRF-estimated activation time series within the Distortion network (inset). (C) Results of the SemNet model, which compares the effects of the LSS-and STRF-estimated activation time series within the Semantic network (inset).

As is clear from Figure 7 and the regression coefficients in Table 2, the broad pattern in the Intelligibility and Distortion networks is the same as that observed in the 'network-within, signal- 
between' models: a strong positive relation to performance in the Intelligibility network and strong negative relation to performance in the Distortion network. One major difference relative to the 'network-within, signal-between' models concerns the Semantic network, which now shows a moderate to strong positive relation with performance. Some other patterns that were suggested by the 'network-within, signal-between' models can be confirmed from the 'networkbetween, signal-within' models. First, in the Intelligibility network, the STRF signals clearly generate better predictions than the LSS signals, regardless of experimental condition. Second, in the Distortion and Semantic networks, performance is better predicted by the LSS signals in the Competing condition and the STRF signals in the Unison condition. This suggests the relation between performance and activation in these networks is mediated entirely by the acoustic distortion introduced by bubbles in the Unison condition, but partially and more strongly mediated by task demands that are not well-captured by bubbles in the Competing condition. In other words, the brain-behavior link in the Distortion and Semantic networks, but not the Intelligibility network, is task-dependent.

Table 2. Regression coefficients and model fit metrics for the 'network-between, signal-within model class.

\begin{tabular}{|c|c|c|c|c|c|}
\hline & \multicolumn{5}{|c|}{ Model Name } \\
\hline & Intel & Distort & Seman & Null & Stacked \\
\hline \multicolumn{6}{|l|}{ Coefficient } \\
\hline $\begin{array}{l}\text { Condition-U } \\
(95 \% \mathrm{CI})\end{array}$ & $\begin{array}{c}0.90 \\
(0.88,0.91)\end{array}$ & $\begin{array}{c}0.91 \\
(0.89,0.93)\end{array}$ & $\begin{array}{c}0.88 \\
(0.86,0.91)\end{array}$ & $\begin{array}{c}0.85 \\
(0.83,0.87)\end{array}$ & -- \\
\hline $\begin{array}{l}\text { Condition-C } \\
(95 \% \mathrm{CI})\end{array}$ & $\begin{array}{c}0.68 \\
(0.67,0.69)\end{array}$ & $\begin{array}{c}0.67 \\
(0.66,0.68)\end{array}$ & $\begin{array}{c}0.67 \\
(0.66,0.68)\end{array}$ & $\begin{array}{c}0.67 \\
(0.66,0.68)\end{array}$ & -- \\
\hline $\begin{array}{l}\text { LSS } \\
(95 \% \mathrm{CI})\end{array}$ & $\begin{array}{c}0.11 \\
(0.02,0.20)\end{array}$ & $\begin{array}{c}-0.37 \\
(-0.44,-0.30)\end{array}$ & $\begin{array}{c}0.11 \\
(0.04,0.17)\end{array}$ & -- & -- \\
\hline $\begin{array}{l}\text { STRF } \\
(95 \% \mathrm{CI})\end{array}$ & $\begin{array}{c}1.07 \\
(0.88,1.26)\end{array}$ & $\begin{array}{c}-0.90 \\
(-1.12,-0.67)\end{array}$ & $\begin{array}{c}0.49 \\
(0.29,0.70)\end{array}$ & -- & -- \\
\hline $\begin{array}{l}\text { Condition:LSS } \\
(95 \% \mathrm{CI})\end{array}$ & $\begin{array}{c}0.00 \\
(-0.06,0.06)\end{array}$ & $\begin{array}{c}-0.02 \\
(-0.07,0.04)\end{array}$ & $\begin{array}{c}-0.04 \\
(-0.10,0.01)\end{array}$ & -- & -- \\
\hline $\begin{array}{l}\text { Condition:STRF } \\
(95 \% \mathrm{CI})\end{array}$ & $\begin{array}{c}0.26 \\
(0.12,0.42)\end{array}$ & $\begin{array}{c}-0.71 \\
(-0.92,-0.51)\end{array}$ & $\begin{array}{c}0.58 \\
(0.37,0.78)\end{array}$ & -- & -- \\
\hline \multicolumn{6}{|l|}{ Model Fit } \\
\hline elpd_loo diff (SE) & 0 & $-62.5(42.5)$ & $-527.8(41.2)$ & $\begin{array}{c}-1004.4 \\
(44.3)\end{array}$ & -- \\
\hline Stacking $w$ & 0.55 & 0.45 & 0 & 0 & -- \\
\hline AUC & 0.76 & 0.75 & 0.7 & 0.64 & 0.77 \\
\hline
\end{tabular}


The model fit metrics in Table 2 show that the Intelligibility network model and Distortion network model perform almost equally in terms of model predictions. The elpd_loo is not significantly different between these models, their stacking weights are both close to 0.5 , and their AUCs are similar. If anything, the Intelligibility network model slightly outperforms the Distortion network model. Both of these models outperform the Semantic network model (lower elpd_loo, zero stacking weight). All three brain-network models outperform the null model.

\subsubsection{All Individual Models}

Outcomes for the 'individual' models are presented in Table 3, which can be interpreted just as Tables 1 and 2. These models represent a single combination of brain network and signal type (e.g., Intel-LSS; Eq. 9). The main motivation for fitting the 'individual' models was to compare the model fit metrics - primarily the elpd_loo and stacking weights - to determine if any single model clearly outperformed the others and whether the stacked combination of models would confirm the patterns suggested by the 'network-within, signal-between' and 'network-between, signal-within' models. In this regard, several interesting patterns were noted. First, the IntelSTRF and Distort-STRF models clearly outperformed all other models. Among these two, the Intel-STRF model significantly outperformed the Distort-STRF model, which indicates that STRF-stimulated activation in the Intelligibility network is the single best predictor of task performance. Second, each of the STRF models outperformed all the LSS models. The likely explanation for this is the strong behavioral predictions obtained from all three brain networks in the Unison condition with the STRF-simulated signal. Finally, the best model combination, as characterized by the stacking weights, relied most strongly on the Intel-STRF model (0.43), followed by the Distort-STRF and Distort-LSS models (0.29 and 0.24, respectively), and then the Intel-LSS model (0.04). These weights essentially recapitulate the 'network-within, signalbetween' weights (roughly three-fourths on STRF vs. LSS) and 'network-between, signal-within' weights (roughly half on the Intelligibility and Distortion networks, and none on the Semantic network), but reveal that the Intelligibility-network predictions are dominated by the STRF signal while the Distortion-network predictions are evenly split between the STRF and LSS signals. Overall, this suggests behavioral performance is best captured by acoustic-phonetic processing in the Intelligibility network and task-modulated, top-down processing in the Distortion network.

Table 3. Regression coefficients and model fit metrics for the 'individual' models.

\begin{tabular}{|c|c|c|c|c|c|c|c|c|}
\hline & \multicolumn{8}{|c|}{ Model Name } \\
\hline & Intel-LSS & Distort-LSS & Seman-LSS & Intel-STRF & $\begin{array}{l}\text { Distort- } \\
\text { STRF }\end{array}$ & $\begin{array}{l}\text { Seman- } \\
\text { STRF }\end{array}$ & Null & Stacked \\
\hline Coefficient & & & & & & & & \\
\hline $\begin{array}{l}\text { Condition-U } \\
(95 \% \mathrm{CI})\end{array}$ & $\begin{array}{c}0.85 \\
(0.83,0.87)\end{array}$ & $\begin{array}{l}0.86 \\
(0.84,0.88)\end{array}$ & $\begin{array}{l}0.85 \\
(0.83,0.87)\end{array}$ & $\begin{array}{l}0.90 \\
(0.88,0.92)\end{array}$ & $\begin{array}{l}0.91 \\
(0.89,0.93)\end{array}$ & $\begin{array}{l}0.88 \\
(0.86,0.91)\end{array}$ & $\begin{array}{l}0.85 \\
(0.83,0.87)\end{array}$ & -- \\
\hline $\begin{array}{l}\text { Condition-C } \\
(95 \% \mathrm{CI})\end{array}$ & $\begin{array}{c}0.67 \\
(0.66,0.68)\end{array}$ & $\begin{array}{l}0.67 \\
(0.66,0.68)\end{array}$ & $\begin{array}{l}0.67 \\
(0.66,0.68)\end{array}$ & $\begin{array}{l}0.68 \\
(0.67,0.69)\end{array}$ & $\begin{array}{l}0.67 \\
(0.66,0.68)\end{array}$ & $\begin{array}{l}0.67 \\
(0.66,0.68)\end{array}$ & $\begin{array}{l}0.67 \\
(0.66,0.68)\end{array}$ & -- \\
\hline
\end{tabular}




\begin{tabular}{|c|c|c|c|c|c|c|c|c|}
\hline $\begin{array}{l}\text { Beta } \\
(95 \% \mathrm{CI})\end{array}$ & $\begin{array}{c}0.37 \\
(0.24,0.50)\end{array}$ & $\begin{array}{l}-0.51 \\
(-0.58,- \\
0.45)\end{array}$ & $\begin{array}{l}0.18 \\
(0.11,0.26)\end{array}$ & $\begin{array}{l}1.12 \\
(0.91,1.34)\end{array}$ & $\begin{array}{l}-1.00 \\
(-1.23,- \\
0.75)\end{array}$ & $\begin{array}{l}0.51 \\
(0.31,0.72)\end{array}$ & -- & -- \\
\hline $\begin{array}{l}\text { Condition:Beta } \\
(95 \% \mathrm{CI})\end{array}$ & $\begin{array}{c}0 \\
(-0.05,0.05)\end{array}$ & $\begin{array}{l}-0.12 \\
(-0.17,- \\
0.07)\end{array}$ & $\begin{array}{l}0.04 \\
(-0.02,0.08)\end{array}$ & $\begin{array}{l}0.27 \\
(0.12,0.43)\end{array}$ & $\begin{array}{l}-0.75 \\
(-0.96,- \\
0.54)\end{array}$ & $\begin{array}{l}0.58 \\
(0.37,0.80)\end{array}$ & -- & -- \\
\hline \multicolumn{9}{|l|}{ Model Fit } \\
\hline $\begin{array}{l}\text { elpd_loo diff } \\
\text { (SE) }\end{array}$ & $\begin{array}{l}-713.3 \\
(48.4)\end{array}$ & $\begin{array}{l}-768.3 \\
(42.1)\end{array}$ & $\begin{array}{l}-938.1 \\
(44.6)\end{array}$ & 0 & $\begin{array}{l}-158.7 \\
(38.4)\end{array}$ & $\begin{array}{l}-524.8 \\
(40.5)\end{array}$ & $\begin{array}{l}-986.4 \\
(44.1)\end{array}$ & -- \\
\hline Stacking $w$ & 0.04 & 0.24 & 0 & 0.43 & 0.29 & 0 & 0 & -- \\
\hline AUC & 0.68 & 0.68 & 0.65 & 0.75 & 0.73 & 0.7 & 0.64 & 0.75 \\
\hline
\end{tabular}

\section{DISCUSSION}

In the present study, we set out to determine: (a) what brain networks are involved in recognition of speech with multiple simultaneous talkers, (b) what speech information is represented in those brain networks, and (c) how activity in those networks relates to performance on a competing speech task. In light of previous research, we expected involvement of both superior temporal and inferior frontal networks, though here we set out to capture differences between these networks in the context of a competing speech task. To this end, we used event-related fMRI and a modified, 3-AFC version of the coordinate response measure (Bolia et al., 2000b) with female and male talkers whose voices were synthesized from the same underlying waveforms using a gender-morph procedure (Venezia et al., 2020). We compared two versions of the task, each with the talkers mixed at $+3 \mathrm{~dB}$ TMR favoring the female talker: Competing, in which the female (target) and male (competing) talkers uttered similar phrases with different keywords, and Unison, in which the female and male talkers uttered identical phrases with the same keywords in perfect synchrony. Spectrotemporalmodulation distortion was applied to the two-talker mixtures using the Auditory Bubbles procedure (Venezia et al., 2016, 2019b), and speech-driven spectrotemporal receptive fields (STRFs) were estimated using a linear encoding model to predict the BOLD signal from the unique patterns of distortion imposed on each trial. We were primarily interested in identifying the brain regions for which encoding model predictions were significantly different across the Competing and Unison conditions. For a given brain region, the presence of such a difference would suggest:

(i) that processing was significantly modulated by trial-to-trial acoustic distortion patterns in one condition but not the other;

(ii) that processing was significantly modulated by trial-to-trial acoustic distortion patterns in both conditions but by different amounts (or more consistently in one condition than the other); or 
(iii) that processing was significantly modulated by trial-to-trial acoustic distortion patterns in both conditions but in different ways (i.e., with different STRF patterns).

Crucially, (ii) and (iii) are not mutually exclusive and (iii) might fail to produce a significant contrast if different STRF patterns result in similarly good predictions of the BOLD signal. $A$ priori, we expected better predictions for Competing than Unison in brain networks that play an important role in recognition of speech with multiple simultaneous talkers.

Initially, we found two large networks for which model predictions (cross-validated $R^{2}$ ) were significantly different across the Competing and Unison conditions. The first includes classic auditory-speech regions in the STG bilaterally (Fig. 4C, yellow; Binder et al., 2000; Poeppel, 2001; Hickok and Poeppel, 2004; Uppenkamp et al., 2006; Bozic et al., 2010). The second includes frontoparietal regions classically involved in cognitive control, working memory, and attention, as well as speech production (Fig. 4C, blue; Vincent et al., 2008; Adank, 2012; Gazzaley and Nobre, 2012; Lückmann et al., 2014; Dixon et al., 2018; Kearney and Guenther, 2019). Model predictions were significantly better in the Competing condition for the STG network and significantly better in the Unison condition for the frontoparietal network. We expected to find significant differences in temporal and frontoparietal regions given the wellknown role of these regions in speech recognition in background noise (Zekveld et al., 2006; Wong et al., 2008, 2009; Golestani et al., 2013; Vaden et al., 2013; Kennedy-Higgins et al., 2020), but the pattern of differences, particularly that predictions were better for Unison than Competing in the frontoparietal network, was unexpected. Indeed, we would have expected better predictions in the Competing condition for both temporal and frontoparietal networks given the relatively higher perceptual and cognitive load in the Competing task. Thus, to explore what was driving the differences in model predictions between conditions, we probed for differences in STRF patterns (encoding model 'weights') between conditions by performing agglomerative clustering on 'mean-difference STRFs' (second-level Competing STRF minus second-level Unison STRF) separately for the STG and frontoparietal networks. For the STG network, only a single cluster was detected (i.e., all cortical surface nodes within the network shared the same STRF pattern; Fig. 5A, red). On the other hand, the frontoparietal network was split into two broad subnetworks according to STRF patterns (Fig. 5A, blue/green).

In the STG network, the STRF pattern in each condition (Unison, Competing) was essentially the same, representing primarily spectrotemporal features that carry phonetic speech content (Figure 5B, top row; see 'bulls-eye' centered at $\sim 4 \mathrm{~Hz}$ at the bottom left of the STRF). Thus, we term this the Intelligibility network. Given the singular STRF pattern observed in the Intelligibility network, we conclude that differences in model predictions between conditions (Competing > Unison) were due to explanation (ii), above: essentially a difference in the predictive 'amplitude' of the STRF rather than a difference in STRF patterns. One of the two frontoparietal subnetworks - located primarily in fronto-opercular regions, the supplementary motor area, and the intraparietal sulcus - demonstrated negative weights in the 'bulls-eye' region of the STRF in the Unison condition (Fig 5B, bottom row, left panel), essentially anticorrelated with the Intelligibility-network STRF; that is, this subnetwork activated more when acoustic-phonetic features that make the speech stream intelligible were absent. In the Competing condition, 
negative STRF weights were observed in the 'bulls-eye' region and on spectrotemporal features associated with the vocal pitch of the (target) female talker, while positive weights were observed on spectrotemporal features associated with the vocal pitch of the (competing) male talker (Fig 5B, bottom row, right panel); in other words, this subnetwork activated more whenever the target speech signal became more distorted, whether due to the absence of phonetic content and/or target vocal pitch or the presence of competing vocal pitch. Thus, we term this the Distortion network. Given the presence of a clear STRF difference between conditions in the Distortion network, we conclude that the difference in model predictions between conditions (Unison > Competing) was due to explanation (iii), above. Finally, the second frontoparietal subnetwork - including classic semantic memory regions in the superior frontal and orbitofrontal cortices, angular and supramarginal gyri, and posterior cingulate cortex - showed similar STRF patterns to the Intelligibility Network, though the STRF in the Competing condition was markedly noisier than in the Unison condition, with a possible addition of positive weight on the vocal pitch of the target talker in the Competing condition (Figure 5B, middle row). Thus, as with the Intelligibility network, we attribute condition differences in model predictions primarily to explanation (ii), above; that is, while this subnetwork activated more to intelligible than unintelligible speech, the amplitude of this difference was greater in the Unison condition than the Competing condition. In light of the strong overlap between this subnetwork and the classic semantic memory network (Binder et al., 2009; Binder and Desai, 2011; Wirth et al., 2011), we term this the Semantic network.

Why, then, were STRF model predictions better for Competing vs. Unison in the Intelligibility network and better for Unison vs. Competing in the Distortion and Semantic networks? For the Intelligibility network, the explanation is straightforward: in the Competing condition, the total amount of phonetic information was larger because each talker produced a different utterance (compared to a single utterance in the Unison condition), and the perceptual 'acoustic-phonetic load' was also greater due to the requirement to segment target phonetic content from competing phonetic content. From the Intelligibility-network STRFs (Figure 5B, top row), we know that predictable fluctuations in activation from trial to trial were primarily related to the relative presence or absence of phonetic content ('bulls-eye' region of the STRF). Since these fluctuations are expected to be larger in the Competing condition (more phonetic content/greater phonetic load), we expect better STRF predictions of the signal. The explanation for the Semantic network is similar. From the Semantic-network STRFs (Figure 5B, middle row), we again know that predictable fluctuations in activation from trial to trial were primarily related to signal intelligibility. If we presume that activation in the Semantic network reflects the maintenance of information in semantic memory (color/number of the keywords) or a semantic search (matching a hypothesized color/number to a stored array of possible color number combinations) prior to generating a response, then we should expect activation only on trials for which the signal is intelligible enough to generate a semantic hypothesis. Thus, when the signal is significantly distorted, no hypothesis is generated and the Semantic network fails to activate regardless of condition; when the signal is not significantly distorted, a hypothesis can be generated and the Semantic network activates. However, in the Competing condition, phonetic interference from the competing talker - which is not well captured by the STRF because phonetic content from the two talkers overlaps - can disrupt hypothesis generation and the 
Semantic network may fail to activate even when the signal is not significantly distorted. In the Unison condition, there should be no such failure of hypothesis generation, so the difference in Semantic-network activation on intelligible vs. unintelligible trials is expected to be larger than in the Competing condition and thus more predictable from the STRF alone.

Finally, in the Distortion network, the pattern of model predictions might be best understood in terms of listening effort (Wild et al., 2012; Peelle, 2018). From the pattern of STRFs in this network (Figure 5B, bottom row), we know the network uniformly activated to distortion from any source, suggesting a compensatory or top-down contribution to task performance. In the Unison condition, the only significant source of distortion was from the spectrotemporal modulation filtering imposed by the Auditory Bubbles procedure, which was well captured by the STRF. However, in the Competing condition, distortion was also introduced by interference from the Competing talker. Some of the competing-talker interference - that related to vocal pitch - was well captured by the STRF, but some - that related to energetic masking in the phonetic content region of the MPS or that related to so-called 'informational masking' (Freyman et al., 2004; Hoen et al., 2007) - was not. Thus, we expect better STRF model predictions in the Unison condition. In terms of listening effort, this implies that, for an acoustically undistorted signal, listening effort is consistently low in the Unison condition, but not necessarily so in the Competing condition where distortion due to informational masking can raise listening effort even in the absence of acoustic distortion. The difference in overall task performance between conditions (Competing $\sim=67 \%$, Unison $\sim=85 \%$ ) is likely also relevant. Recall that the overall Therefore, on trials with a relatively large amount of signal distortion, the probability of outright guessing was likely higher in the Competing condition than the Unison condition. In terms of listening effort, we generally expect effort to increase with increasing distortion, but this relationship is non-monotonic; eventually, the signal becomes so distorted that the perceived likelihood of solving the task falls below the probability of guessing correctly, and listening effort decreases. We suggest that, at $67 \%$ overall performance, trials in the Competing condition were more likely to occupy this falling portion of the listening effort function. Thus, the relationship between listening effort and amount of spectrotemporal distortion was weaker for Competing vs. Unison when distortion was low (due to informational masking) and when distortion was high (due to increased guessing), with each of these effects contributing to relatively poorer STRF model predictions in the Competing condition.

Overall, these results suggest that the Intelligibility network performs bottom-up extraction of phonetic content, while the Distortion network performs top-down analysis of distorted speech that scales with listening effort, and the Semantic network mediates semantic hypotheses, the generation of which depends completely on successful extraction of task-relevant information from the target speech stream. Below, we explore the roles of these networks in greater detail including the relation between network activation and behavioral performance as quantified presently via 'neurometric' functions. 
1274

1275

1276

1277

1278

1279

1280

1281

1282

1283

1284

1285

1286

1287

1288

1289

1290

1291

1292

1293

1294

1295

1296

1297

1298

1299

1300

1301

1302

1303

1304

1305

1306

1307

1308

1309

1310

1311

1312

1313

1314

1315

1316

1317

\subsection{Relative Contributions of Intelligibility and Distortion Networks to Behavioral Performance}

Overall, the results of neurometric function modeling support the conclusion that the Intelligibility network contributes to behavior in a bottom-up fashion while the Distortion network contributes to behavior in a top-down fashion. First, and most straightforwardly, the slope of the neurometric function tended to be positive (more activation = better performance) in the Intelligibility network and negative (more activation $=$ worse performance) in the Distortion network (Figs. 6-7, top two rows; Tables 1-3, sign of Coefficient). Second, the positive association with behavior in the Intelligibility network was very well captured by its STRF, as indicated by similar neurometricfunction slopes in the Intelligibility network for the LSS and STRF models in the "network-within, signal-between' model class (Figure 6, top row), larger neurometric function slopes for the STRF model than the LSS model in the 'network-between, signal-within' model class (Figure 7, top row), and better model predictive performance (AUC, elpd_loo, stacking weight) for the STRF-individual model vs. the LSS-individual model in the Intelligibility network (Table 3). Neurometric-function slopes were also similarly steep across the Competing and Unison conditions in the Intelligibility network for the LSS model (Table 2, Condition:LSS x Intel), suggesting the network exerted a comparable influence on performance regardless of the task demands. The neurometric-function slope was slightly larger in the Unison condition than the Competing condition for the STRF model (Table 2, Condition:STRF x Intel), but this likely reflects the difference in overall task difficulty between conditions - specifically, trials were more likely to be clearly intelligible or clearly unintelligible in the Unison condition, leading to a stronger correlation between behavioral performance and STRF-simulated brain activity, whereas the STRFs themselves were highly correlated with behavioral classification images for intelligibility (Eq. 1, Figure 3B). There was some evidence for contributions to behavior beyond the STRF in the Intelligibility network, including a significantly non-zero slope for the LSS model in the 'network-between, signal-within' model class (Table 2, LSS x Intel) and a non-zero stacking weight on the LSS-individual model in the Intelligibility network (Table 3,w $x$ Intel-LSS), but these contributions were of a negligible magnitude compared to STRF-mediated contributions (Table 2, STRF $x$ Intel; Table 3, wx Intel-STRF).

Finally, the negative association with behavior in the Distortion network was not entirely well captured by its STRF, although this was true only in the Competing condition. Specifically, the Competing neurometric-function slope was reduced for the STRF model compared to the LSS model in the 'network-between, signal-within' model class (Figure 7, second row, red). This is consistent with our suggestion above that, in the Competing condition, sources of distortion that are not well captured by the STRF (e.g., informational masking) significantly modulate brain activation in the Distortion network; indeed, these sources of distortion also modulate behavior and thus behavior is better predicted by the LSS model than the STRF model in the Competing condition. Interestingly, the reverse pattern was observed in the Distortion network for the Unison condition - namely, the neurometric-function slope was steeper for the STRF model than the LSS model in the 'network-between, signal-within' model class (Fig. 7, second row, blue). In the Unison condition, there was only a single source of distortion - that induced by bubbles filtering - and this was very well captured by the STRF, thus leading to stronger predictions of behavior by the STRF model. This pattern of better predictions in the Distortion 
network for the STRF model in the Unison condition and the LSS model in the Competing condition are reflected in the stacking weights for the individual models (Table 3, Distort-LSS = 0.24 , Distort-STRF $=0.25$ ), which suggest that STRF- and non-STRF-mediated sources of activation contribute roughly equally to predictions of behavioral performance. Broadly, this appears to be consistent with the "frontal-cognitive" perspective on top-down contributions to speech perception in background noise, wherein the Distortion network mediates behavior via domain general "multiple-demand" processes (Blank et al., 2014; Noyce et al., 2017; Mineroff et al., 2018; Diachek et al., 2020). However, given our suggestion above that condition differences in STRF model performance primarily reflect differences in the relative listening effort required for trials with high vs. low levels of extrinsic distortion due to bubbles, we cannot rule out a "frontal-motor" (Skipper et al., 2007; Bever and Poeppel, 2010; Schwartz et al., 2012; Cope et al., 2017) explanation of the neurometric-function model results. The logic here is as follows. In the Competing condition, listening effort is less predictably related to the level of extrinsic distortion imposed by bubbles, and therefore is better captured by the LSS model than the STRF model. Assuming listening effort is strongly correlated with behavioral performance, the LSS model therefore performs better at predicting behavioral performance. If we further assume that an increase in listening effort from any source (e.g., bubbles distortion, informational masking, etc.) leads to an increased contribution from top-down, speech-motor predictions, then the results are consistent with the "frontal-motor" point of view. Since these assumptions must hold in order for the "frontal-motor" view to be supported, the "frontal-cognitive" view provides the more parsimonious account of the present data - i.e., activation in multiple-demand networks is expected to scale with effort by definition.

Given this clear support for a bottom-up vs. top-down dichotomy relative to the contributions to behavior from the Intelligibility and Distortion networks, respectively, a remaining issue concerns the relative magnitudes and level of interdependence of these contributions. Several pieces of evidence suggest the contributions to behavioral performance from these networks were roughly equal in terms of magnitude and at least partially independent. The first comes from the neurometric-function slopes in the 'network-within, signal-between' class of models. Here, the networks must compete with one another to explain trial-to-trial fluctuations in behavioral performance. For this class of models, although the neurometric functions in the Intelligibility and Distortion networks had opposing signs, the slopes were essentially equal in magnitude when averaged across the LSS and STRF models. This suggests that activation in the Intelligibility and Distortion networks captured different components of behavioral performance but made similarly important contributions to performance. Second, for the 'network-between, signal-within' class of models where cross-model comparisons (elpd_loo, stacking weights) reflect the relative contribution of each network to behavioral performance, the Intelligibility and Distortion networks were roughly equivalent (Table 2, elpd_loo diff $<2$ SEs, $w=0.55,0.45$, for Intelligibility and Distortion, respectively). Similarly, the individual model weights (Table 3) were nearly equivalent for the Intelligibility (0.47) and Distortion (0.53) networks when summed across LSS and STRF models. Again, these results suggest that, considering the STRF- and non-STRF-related avenues through which these networks may mediate behavioral performance, their contributions were similar in magnitude and not completely dependent on one another. In other words, it was not simply the case that top-down networks were engaged 
1362

whenever bottom-up processing failed or top-down networks were not engaged whenever bottom-processing succeeded. Rather, a more consistent interpretation of the data is that bottom-up and top-down contributions to behavior varied considerably within the two classes of behavioral responses (correct, incorrect).

For example, imagine a trial in the Competing condition for which the phonetic content of the signal is relatively uncorrupted by bubbles filtering. Here, we expect activation in the Intelligibility network to be relatively high, and thus our neurometric function in the Intelligibility network will predict that the listener is likely to select the correct response. However, let us further suppose that the listener experiences a large amount of informational masking on the same trial, ultimately selecting the incorrect response. Here, we also expect activation in the Distortion network to be high due to the informational masking, and our neurometric function in the Distortion network will (correctly) predict that the listener is likely to select the incorrect response. Now imagine a trial in either the Unison or Competing condition for which the signal is severely corrupted by bubbles filtering. Here, we expect activation in the Distortion network to be relatively low because the signal is so distorted that the listener resorts to guessing. Thus, the neurometric function in the distortion network predicts a high likelihood of responding correctly. We also expect activation in the Intelligibility network to be low, but in this case the neurometric function predicts a low likelihood of responding correctly. Since the probability of guessing correctly is only $\mathbf{0 . 3 3}$, the signal in the Intelligibility network will be a more reliable predictor of performance, capturing the 0.67 probability of failing to guess correctly. Taken as a whole, we see that in some scenarios the signal in the Distortion network is a better predictor of behavior, while in other scenarios the signal in the Intelligibility network is the better predictor of behavior. Thus, to make the best possible predictions across all scenarios, the neurometricfunction model should place some weight on the Intelligibility network and some weight on the Distortion network, which reflects the constant and dynamic interplay between these networks regardless of whether the behavioral response is ultimately correct or incorrect.

\subsection{Contribution of Semantic Network to Behavioral Performance}

Broadly, activation in the Semantic network was positively correlated with behavioral performance on the 3-AFC coordinate response measure task. However, this contribution to behavioral performance appeared to be entirely dependent on the output of the Intelligibility and Distortion networks. Specifically, when the Semantic network was made to compete directly with the Intelligibility and Distortion networks to explain behavior in the 'network-within, signalbetween' class of models, the neurometric function slope in the Semantic network was essentially zero (Figure 6). Moreover, in the 'network-between, signal-within' class of models, the stacking weight on the Semantic network was precisely zero. There was a positive association between STRF-simulated activation in the Semantic network and behavioral performance (Figure 7), dominated by the Unison condition (Table 2, STRF/condition:STRF), but a zero stacking weight suggests this STRF-mediated effect was entirely superseded by the positive STRF-mediated effect in the Intelligibility network. Finally, there was a positive association between activation in the Semantic network and behavioral performance for both the Seman-LSS and Seman-STRF models within the 'individual models' (Table 3, Beta), but again the stacking weight on these models was precisely zero. Taken together, and considering 
our earlier characterization of the functional role of the Semantic network, these findings suggest that generation and maintenance of semantic hypotheses in the Semantic network leads to better performance on the task, but the ability to generate such hypotheses depends entirely on effective extraction of the information in the speech signal by the Intelligibility and Distortion networks.

This is somewhat unsurprising in the context of the present experiment. Indeed, the items in the coordinate response measure corpus by design provide little to no semantic constraint (Bolia et al., 2000b). Thus, we might expect a more substantial role for the Semantic network in perception of naturalistic, narrative speech, for which buildup of semantic context has been shown to play an early and important role in word recognition (Nieuwland and Van Berkum, 2006; Pulvermüller et al., 2009; Boudewyn et al., 2012, 2019). Moreover, relative to the Distortion and Intelligibility networks, the Semantic network's contribution to recognition performance may depend more on the characteristics of the listener, since semantic context has been shown to play an outsize role in speech recognition for aging listeners and listeners with hearing loss (Gordon-Salant and Fitzgibbons, 1997; Sommers and Danielson, 1999; Benichov et al., 2012; Smayda et al., 2016; Holmes et al., 2018; Koeritzer et al., 2018). Interestingly, recent work shows that activity in the Semantic network is associated with semantic predictability, though negatively so, which suggests this network is recruited to retrieve semantic information when it is less predictable (Carter et al., 2019). Thus, the semantic network may play a role in compensating for degraded speech input (Golestani et al., 2013), especially when semantic predictability is also low.

\section{SUMMARY/CONCLUSION}

We identified three cortical networks involved in the perception of speech with multiple simultaneous talkers: (i) an Intelligibility network in the bilateral superior temporal gyrus/sulcus, which responded most prominently to phonetic speech content and had a positive association with speech recognition performance; (ii) a Distortion network in the bilateral inferior and middle frontal lobes, supplementary motor areas, and the left inferior parietal lobe, which responded most prominently to signal corruption from any source (absence of phonetic content or target talker pitch, presence of competing talker pitch) and had a negative association with speech recognition performance; and (iii) a Semantic network in the bilateral ventromedial prefrontal cortex, left posterior cingulate and dorsal prefrontal cortices, and left angular/supramarginal gyri, which responded most prominently to phonetic speech content and had a weak positive association with speech recognition performance. Comparison of speech-driven STRFs in the Intelligibility and Distortion networks suggest that the Intelligibility network plays a bottom-up, non-task-specific role in supporting speech recognition, while the Distortion network plays a topdown, task-specific role in supporting speech recognition. Neurometric function modeling showed that the influence of the Intelligibility network on speech recognition performance was entirely STRF-mediated, while the influence of the Distortion network on performance was mediated by sources of distortion within (e.g., signal corruption, competing talker vocal pitch) and outside (e.g., informational masking) the STRF domain, which supports a more domain general or "frontal-cognitive" characterization of the Distortion network in which network activation scales with listening effort. Additionally, the contributions to speech recognition 
performance from the Intelligibility and Distortion networks were roughly equal in magnitude and at least partially independent, and these contributions entirely superseded those of the Semantic network, which suggests the Semantic network is situated at a higher level of processing that, in the context of the present study, depends entirely on the successful extraction of speech information by the Intelligibility and Distortion networks. Overall, these findings suggest a constant and dynamic interplay between the Intelligibility and Distortion networks, the outcome of which determines the engagement of the Semantic network. Future work should determine how the relative contributions and dependencies among these three networks are modulated by the speech materials (e.g., naturalistic and/or context-constrained versus contrived and/or context free), number of competing talkers (e.g., one versus multiple), and characteristics of individual listeners (e.g., age, hearing status, and cognitive ability).

\section{ACKNOWLEDGEMENTS}

This work was supported by the U.S. Department of Veterans Affairs, Veterans Health Administration, Rehabilitation Research \& Development Service Award IK2RX002702 to J.H.V. This material is the result of work supported with resources from and the use of facilities at the VA Loma Linda Healthcare System, Loma Linda, CA. The contents do not represent the views of the U.S. Department of Veterans Affairs or the U.S. government.

\section{DATA AND CODE AVAILABILITY}

All code used for stimulus generation, presentation, and data analysis can be found at found at https://osf.io/ftmpa/. This repository also contains the results of second-level fMRI analyses in cortical surface format (text files that can be mapped to the cortical surface using SUMA) as well as the results of neurometric function analyses ( $R$ data files). Code for generating the final plots is available in the repository where applicable. In accordance with VA policy, interested parties may obtain the raw MRI and behavioral data only once a Data Use Agreement has been finalized between the requesting institution and the VA Loma Linda Healthcare System, U.S. Department of Veterans Affairs.

\section{REFERENCES}

Adank P (2012) The neural bases of difficult speech comprehension and speech production: Two Activation Likelihood Estimation (ALE) meta-analyses. Brain Lang 122:42-54.

Arlinger S, Lunner T, Lyxell B, Kathleen Pichora-Fuller M (2009) The emergence of cognitive hearing science. Scand J Psychol 50:371-384.

Benichov J, Cox LC, Tun PA, Wingfield A (2012) Word recognition within a linguistic context: effects of age, hearing acuity, verbal ability, and cognitive function. Ear Hear 33:250-256.

Bever TG, Poeppel D (2010) Analysis by synthesis: a (re-) emerging program of research for language and vision. Biolinguistics 4:174-200. 
Binder JR, Desai RH (2011) The neurobiology of semantic memory. Trends Cogn Sci 15:527536.

Binder JR, Desai RH, Graves WW, Conant LL (2009) Where is the semantic system? A critical review and meta-analysis of 120 functional neuroimaging studies. Cereb Cortex 19:27672796.

Binder JR, Frost JA, Hammeke TA, Bellgowan PSF, Springer JA, Kaufman JN, Possing ET (2000) Human temporal lobe activation by speech and nonspeech sounds. Cereb Cortex 10:512-528.

Binder JR, Liebenthal E, Possing ET, Medler DA, Ward BD (2004) Neural correlates of sensory and decision processes in auditory object identification. Nat Neurosci 7:295-301.

Blank I, Kanwisher N, Fedorenko E (2014) A functional dissociation between language and multiple-demand systems revealed in patterns of BOLD signal fluctuations. J Neurophysiol 112:1105-1118.

Boersma P (2001) Praat, a system for doing phonetics by computer. Glot Int Available at: https://ci.nii.ac.jp/naid/10026090047/.

Bolia RS, Nelson WT, Ericson MA, Simpson BD (2000a) A speech corpus for multitalker communications research. J Acoust Soc Am 107:1065-1066.

Bolia RS, Nelson WT, Ericson MA, Simpson BD (2000b) A speech corpus for multitalker communications research. J Acoust Soc Am 107:1065-1066.

Boudewyn MA, Blalock AR, Long DL, Swaab TY (2019) Adaptation to Animacy Violations during Listening Comprehension. Cogn Affect Behav Neurosci 19:1247-1258.

Boudewyn MA, Gordon PC, Long D, Polse L, Swaab TY (2012) Does Discourse Congruence Influence Spoken Language Comprehension before Lexical Association? Evidence from Event-Related Potentials. Lang Cogn Process 27:698-733.

Bozic M, Tyler LK, Ives DT, Randall B, Marslen-Wilson WD (2010) Bihemispheric foundations for human speech comprehension. Proc Natl Acad Sci U S A 107:17439-17444.

Brodbeck C, Presacco A, Simon JZ (2018) Neural source dynamics of brain responses to continuous stimuli: Speech processing from acoustics to comprehension. Neuroimage 172:162-174.

Buchsbaum BR, Baldo J, Okada K, Berman KF, Dronkers N, D’Esposito M, Hickok G (2011) Conduction aphasia, sensory-motor integration, and phonological short-term memory - An aggregate analysis of lesion and fMRI data. Brain Lang 119:119-128.

Bürkner PC (2017) brms: An R package for Bayesian multilevel models using Stan. J Stat Softw Available at: https://www.jstatsoft.org/index.php/jss/article/view/v080i01.

Carter BT, Foster B, Muncy NM, Luke SG (2019) Linguistic networks associated with lexical, semantic and syntactic predictability in reading: A fixation-related fMRI study. Neuroimage 189:224-240.

Cauley SF, Polimeni JR, Bhat H, Wald LL, Setsompop K (2014) Interslice leakage artifact 
reduction technique for simultaneous multislice acquisitions. Magn Reson Med 72:93-102.

Cherry EC (1953) Some experiments on the recognition of speech, with one and with two ears. J Acoust Soc Am 25:975-979.

Cope TE, Sohoglu E, Sedley W, Patterson K, Jones PS, Wiggins J, Dawson C, Grube M, Carlyon RP, Griffiths TD, Davis MH, Rowe JB (2017) Evidence for causal top-down frontal contributions to predictive processes in speech perception. Nat Commun 8:2154.

Damasio AR, Geschwind N (1984) The neural basis of language. Annu Rev Neurosci 7:127147.

Damasio H, Tranel D, Grabowski T, Adolphs R, Damasio A (2004) Neural systems behind word and concept retrieval. Cognition 92:179-229.

D'Ausilio A, Craighero L, Fadiga L (2012) The contribution of the frontal lobe to the perception of speech. J Neurolinguistics 25:328-335.

David SV, Mesgarani N, Shamma SA (2007) Estimating sparse spectro-temporal receptive fields with natural stimuli. Network 18:191-212.

de Heer WA, Huth AG, Griffiths TL, Gallant JL, Theunissen FE (2017) The Hierarchical Cortical Organization of Human Speech Processing. J Neurosci 37:6539-6557.

Diachek E, Blank I, Siegelman M, Affourtit J, Fedorenko E (2020) The Domain-General Multiple Demand (MD) Network Does Not Support Core Aspects of Language Comprehension: A Large-Scale fMRI Investigation. J Neurosci 40:4536-4550.

Diehl RL, Lotto AJ, Holt LL (2004) Speech perception. Annu Rev Psychol 55:149-179.

Dixon ML, De La Vega A, Mills C, Andrews-Hanna J, Spreng RN, Cole MW, Christoff K (2018) Heterogeneity within the frontoparietal control network and its relationship to the default and dorsal attention networks. Proc Natl Acad Sci U S A 115:E1598-E1607.

Du Y, Buchsbaum BR, Grady CL, Alain C (2016) Increased activity in frontal motor cortex compensates impaired speech perception in older adults. Nat Commun 7:12241.

Eklund A, Nichols TE, Knutsson H (2016) Cluster failure: Why fMRI inferences for spatial extent have inflated false-positive rates. Proc Natl Acad Sci U S A 113:7900-7905.

Erb J, Henry MJ, Eisner F, Obleser J (2013) The brain dynamics of rapid perceptual adaptation to adverse listening conditions. J Neurosci 33:10688-10697.

Erb J, Obleser J (2013) Upregulation of cognitive control networks in older adults' speech comprehension. Front Syst Neurosci 7:116.

Fiedler L, Wöstmann M, Herbst SK, Obleser J (2019) Late cortical tracking of ignored speech facilitates neural selectivity in acoustically challenging conditions. Neuroimage 186:33-42.

Folstein MF, Folstein SE, McHugh PR (1975) "Mini-mental state": a practical method for grading the cognitive state of patients for the clinician. Journal of psychiatric Available at: https://www.academia.edu/download/48794085/0022-3956_2875_2990026-62016091328497-y2c5d5.pdf. 
1566

1567

1568

1569

1570

1571

1572

1573

1574

1575

1576

1577

1578

1579

1580

1581

1582

1583

1584

1585

1586

1587

1588

1589

1590

1591

1592

1593

1594

1595

1596

1597

1598

1599

1600

1601

1602

1603

Freyman RL, Balakrishnan U, Helfer KS (2004) Effect of number of masking talkers and auditory priming on informational masking in speech recognition. J Acoust Soc Am 115:2246-2256.

Galantucci B, Fowler CA, Turvey MT (2006) The motor theory of speech perception reviewed. Psychon Bull Rev 13:361-377.

Gao Y, Wang Q, Ding Y, Wang C, Li H, Wu X, Qu T, Li L (2017) Selective Attention Enhances Beta-Band Cortical Oscillation to Speech under "Cocktail-Party" Listening Conditions. Front Hum Neurosci 11:34.

Gazzaley A, Nobre AC (2012) Top-down modulation: bridging selective attention and working memory. Trends Cogn Sci 16:129-135.

Gernsbacher MA, Kaschak MP (2003) Neuroimaging studies of language production and comprehension. Annu Rev Psychol 54:91-114.

Glover GH (1999) Deconvolution of impulse response in event-related BOLD fMRI1. Neuroimage Available at: https://www.sciencedirect.com/science/article/pii/S1053811998904190.

Golestani N, Hervais-Adelman A, Obleser J, Scott SK (2013) Semantic versus perceptual interactions in neural processing of speech-in-noise. Neuroimage 79:52-61.

Golumbic EMZ, Ding N, Bickel S, Lakatos P, Schevon CA, McKhann GM, Goodman RR, Emerson R, Mehta AD, Simon JZ (2013) Mechanisms underlying selective neuronal tracking of attended speech at a "cocktail party." Neuron 77:980-991.

Gordon-Salant S, Fitzgibbons PJ (1997) Selected cognitive factors and speech recognition performance among young and elderly listeners. J Speech Lang Hear Res 40:423-431.

Hickok G (2014) The architecture of speech production and the role of the phoneme in speech processing. Lang Cogn Process 29:2-20.

Hickok G, Poeppel D (2000) Towards a functional neuroanatomy of speech perception. Trends Cogn Sci 4:131-138.

Hickok G, Poeppel D (2004) Dorsal and ventral streams: a framework for understanding aspects of the functional anatomy of language. Cognition 92:67-99.

Hickok G, Poeppel D (2007) The cortical organization of speech processing. Nat Rev Neurosci 8:393-402.

Hoen M, Meunier F, Grataloup C-L, Pellegrino F, Grimault N, Perrin F, Perrot X, Collet L (2007) Phonetic and lexical interferences in informational masking during speech-in-speech comprehension. Speech Commun 49:905-916.

Hoffman MD, Gelman A (2014) The No-U-Turn sampler: adaptively setting path lengths in Hamiltonian Monte Carlo. J Mach Learn Res Available at: https://www.jmlr.org/papers/volume15/hoffman14a/hoffman14a.pdf.

Holmes E, Folkeard P, Johnsrude IS, Scollie S (2018) Semantic context improves speech intelligibility and reduces listening effort for listeners with hearing impairment. Int $J$ Audiol 
Holt LL, Lotto AJ (2008) Speech perception within an auditory cognitive science framework. Curr Dir Psychol Sci 17:42-46.

Jacquemot C, Scott SK (2006) What is the relationship between phonological short-term memory and speech processing? Trends Cogn Sci 10:480-486.

Kaernbach C (1991) Simple adaptive testing with the weighted up-down method. Percept Psychophys 49:227-229.

Kearney E, Guenther FH (2019) Articulating: The Neural Mechanisms of Speech Production. Lang Cogn Neurosci 34:1214-1229.

Kennedy-Higgins D, Devlin JT, Nuttall HE, Adank P (2020) The Causal Role of Left and Right Superior Temporal Gyri in Speech Perception in Noise: A Transcranial Magnetic Stimulation Study. J Cogn Neurosci 32:1092-1103.

Kleiner M, Brainard D, Pelli D, Ingling A, Murray R, Broussard C, Others (2007) What's new in Psychtoolbox-3. Perception 36:1-1.

Koeritzer MA, Rogers CS, Van Engen KJ, Peelle JE (2018) The Impact of Age, Background Noise, Semantic Ambiguity, and Hearing Loss on Recognition Memory for Spoken Sentences. J Speech Lang Hear Res 61:740-751.

Liberman AM (1957) Some results of research on speech perception. J Acoust Soc Am 29:117123.

Liberman AM, Mattingly IG (1985) The motor theory of speech perception revised. Cognition 21:1-36.

Lotto AJ, Hickok GS, Holt LL (2009) Reflections on mirror neurons and speech perception. Trends Cogn Sci 13:110-114.

Lückmann HC, Jacobs HIL, Sack AT (2014) The cross-functional role of frontoparietal regions in cognition: internal attention as the overarching mechanism. Prog Neurobiol 116:66-86.

Mattys SL, Davis MH, Bradlow AR, Scott SK (2012) Speech recognition in adverse conditions: A review. Lang Cogn Process 27:953-978.

Mineroff Z, Blank IA, Mahowald K, Fedorenko E (2018) A robust dissociation among the language, multiple demand, and default mode networks: Evidence from inter-region correlations in effect size. Neuropsychologia 119:501-511.

Mumford JA, Turner BO, Ashby FG, Poldrack RA (2012) Deconvolving BOLD activation in event-related designs for multivoxel pattern classification analyses. Neuroimage 59:26362643.

Nieuwland MS, Van Berkum JJA (2006) When peanuts fall in love: N400 evidence for the power of discourse. J Cogn Neurosci 18:1098-1111.

Noyce AL, Cestero N, Michalka SW, Shinn-Cunningham BG, Somers DC (2017) SensoryBiased and Multiple-Demand Processing in Human Lateral Frontal Cortex. J Neurosci 
Oosterhof NN, Connolly AC, Haxby JV (2016) CoSMoMVPA: Multi-Modal Multivariate Pattern Analysis of Neuroimaging Data in Matlab/GNU Octave. Front Neuroinform 10:27.

1644 1645
Oosterhof NN, Wiestler T, Downing PE, Diedrichsen J (2011) A comparison of volume-based and surface-based multi-voxel pattern analysis. Neuroimage 56:593-600.

Peelle JE (2018) Listening Effort: How the Cognitive Consequences of Acoustic Challenge Are Reflected in Brain and Behavior. Ear Hear 39:204-214.

Petrie EC, Cross DJ, Yarnykh VL, Richards T, Martin NM, Pagulayan K, Hoff D, Hart K, Mayer C, Tarabochia M, Raskind MA, Minoshima S, Peskind ER (2014) Neuroimaging, behavioral, and psychological sequelae of repetitive combined blast/impact mild traumatic brain injury in Iraq and Afghanistan war veterans. J Neurotrauma 31:425-436.

Pichora-Fuller MK, Kramer SE, Eckert MA, Edwards B, Hornsby BWY, Humes LE, Lemke U, Lunner T, Matthen M, Mackersie CL (2016) Hearing impairment and cognitive energy: The framework for understanding effortful listening (FUEL). Ear Hear 37:5S - 27S.

Poeppel D (2001) Pure word deafness and the bilateral processing of the speech code. Cogn Sci 25:679-693.

Průša Z, Balazs P (2017) A noniterative method for reconstruction of phase from STFT magnitude. IEEE/ACM Transactions on Available at: https://ieeexplore.ieee.org/abstract/document/7890450/.

Pulvermuller F, Fadiga L (2010) Active perception: sensorimotor circuits as a cortical basis for language. Nat Rev Neurosci 11:351-360.

Pulvermüller F, Shtyrov Y, Hauk O (2009) Understanding in an instant: neurophysiological evidence for mechanistic language circuits in the brain. Brain Lang 110:81-94.

Puschmann S, Baillet S, Zatorre RJ (2019) Musicians at the Cocktail Party: Neural Substrates of Musical Training During Selective Listening in Multispeaker Situations. Cereb Cortex 29:3253-3265.

Puschmann S, Steinkamp S, Gillich I, Mirkovic B, Debener S, Thiel CM (2017) The Right Temporoparietal Junction Supports Speech Tracking During Selective Listening: Evidence from Concurrent EEG-fMRI. J Neurosci 37:11505-11516.

Rimmele JM, Zion Golumbic E, Schröger E, Poeppel D (2015) The effects of selective attention and speech acoustics on neural speech-tracking in a multi-talker scene. Cortex 68:144154.

Rizzolatti G, Craighero L (2004) The mirror-neuron system. Annu Rev Neurosci 27:169-192.

Rönnberg J, Rudner M, Lunner T (2011) Cognitive hearing science: The legacy of Stuart Gatehouse. Trends Amplif 15:140-148.

Rousseeuw PJ (1987) Silhouettes: a graphical aid to the interpretation and validation of cluster analysis. J Comput Appl Math Available at: https://www.sciencedirect.com/science/article/pii/0377042787901257. 
Schwartz J-L, Basirat A, Ménard L, Sato M (2012) The Perception-for-Action-Control Theory (PACT): A perceptuo-motor theory of speech perception. J Neurolinguistics 25:336-354.

Scott SK, McGettigan C, Eisner F (2009) A little more conversation, a little less action-candidate roles for the motor cortex in speech perception. Nat Rev Neurosci 10:295-302.

Scott TL, Perrachione TK (2019) Common cortical architectures for phonological working memory identified in individual brains. Neuroimage 202:116096.

Skipper Jl, van Wassenhove V, Nusbaum HC, Small SL (2007) Hearing lips and seeing voices: how cortical areas supporting speech production mediate audiovisual speech perception. Cereb Cortex 17:2387-2399.

Smayda KE, Van Engen KJ, Maddox WT, Chandrasekaran B (2016) Audio-Visual and Meaningful Semantic Context Enhancements in Older and Younger Adults. PLoS One 11:e0152773.

Smith SM, Beckmann CF, Andersson J, Auerbach EJ (2013) Resting-state fMRI in the human connectome project. Neuroimage Available at: https://www.sciencedirect.com/science/article/pii/S1053811913005338.

Smith SM, Nichols TE (2009) Threshold-free cluster enhancement: addressing problems of smoothing, threshold dependence and localisation in cluster inference. Neuroimage 44:8398.

Sommers MS, Danielson SM (1999) Inhibitory processes and spoken word recognition in young and older adults: the interaction of lexical competition and semantic context. Psychol Aging 14:458-472.

Stokes RC, Venezia JH, Hickok G (2019) The motor system's [modest] contribution to speech perception. Psychon Bull Rev 26:1354-1366.

Uppenkamp S, Johnsrude IS, Norris D, Marslen-Wilson W, Patterson RD (2006) Locating the initial stages of speech-sound processing in human temporal cortex. Neuroimage 31:12841296.

Vaden KI Jr, Kuchinsky SE, Ahlstrom JB, Dubno JR, Eckert MA (2015) Cortical activity predicts which older adults recognize speech in noise and when. J Neurosci 35:3929-3937.

Vaden KI Jr, Kuchinsky SE, Cute SL, Ahlstrom JB, Dubno JR, Eckert MA (2013) The cinguloopercular network provides word-recognition benefit. J Neurosci 33:18979-18986.

Vaden KI Jr, Teubner-Rhodes S, Ahlstrom JB, Dubno JR, Eckert MA (2017) Cingulo-opercular activity affects incidental memory encoding for speech in noise. Neuroimage 157:381-387.

Vaden KI, Kuchinsky SE, Ahlstrom JB, Teubner-Rhodes SE, Dubno JR, Eckert MA (2016) Cingulo-opercular function during word recognition in noise for older adults with hearing loss. Exp Aging Res 42:67-82.

Vehtari A, Gelman A, Gabry J (2017) Practical Bayesian model evaluation using leave-one-out cross-validation and WAIC. Stat Comput Available at: https://idp.springer.com/authorize/casa?redirect_uri=https://link.springer.com/article/10.100 7/s11222-016-9696- 
4\&casa_token=yDh7VC8PigAAAAAA:PyUeudhAvO5p_1PEPoN3uW7R1EiCvdtzrV7CbUSr gNVXSD-o_BqwekUE_PTHdMMPquFL3bcSG5I3MhhK.

Venezia J (2019) Data-driven methods in a model-driven world: Making sense of auditory classification images for continuous speech. J Acoust Soc Am 145:1685-1685.

Venezia JH, Hickok G (2009) Mirror Neurons, the Motor System and Language: From the Motor Theory to Embodied Cognition and Beyond. Lang Linguist Compass 3:1403-1416.

Venezia JH, Hickok G, Richards VM (2016) Auditory “bubbles": Efficient classification of the spectrotemporal modulations essential for speech intelligibility. J Acoust Soc Am 140:1072.

Venezia JH, Leek MR, Lindeman MP (2020) Suprathreshold Differences in Competing Speech Perception in Older Listeners With Normal and Impaired Hearing. J Speech Lang Hear Res 63:2141-2161.

Venezia JH, Martin A-G, Hickok G, Richards VM (2019a) Identification of the Spectrotemporal Modulations That Support Speech Intelligibility in Hearing-Impaired and Normal-Hearing Listeners. J Speech Lang Hear Res 62:1051-1067.

Venezia JH, Saberi K, Chubb C, Hickok G (2012) Response bias modulates the speech motor system during syllable discrimination. Front Psychol 3 Available at: http://www.frontiersin.org/Journal/Abstract.aspx?s=86\&name=auditory_cognitive_neuroscie nce\&ART_DOI=10.3389/fpsyg.2012.00157.

Venezia JH, Thurman SM, Richards VM, Hickok G (2019b) Hierarchy of speech-driven spectrotemporal receptive fields in human auditory cortex. Neuroimage 186:647-666.

Venezia J, Leek M (2020) Suprathreshold differences in competing speech perception in older listeners. Journal of Speech, Language, and Hearing Research.

Vincent JL, Kahn I, Snyder AZ, Raichle ME, Buckner RL (2008) Evidence for a frontoparietal control system revealed by intrinsic functional connectivity. J Neurophysiol 100:3328-3342.

Whitfield-Gabrieli S, Nieto-Castanon A (2012) Conn: A Functional Connectivity Toolbox for Correlated and Anticorrelated Brain Networks. Brain Connect Available at: https://www.liebertpub.com/doi/abs/10.1089/brain.2012.0073.

Wild CJ, Yusuf A, Wilson DE, Peelle JE, Davis MH, Johnsrude IS (2012) Effortful listening: the processing of degraded speech depends critically on attention. J Neurosci 32:1401014021.

Wilkins KC, Lang AJ, Norman SB (2011) Synthesis of the psychometric properties of the PTSD checklist (PCL) military, civilian, and specific versions. Depress Anxiety 28:596-606.

Wirth M, Jann K, Dierks T, Federspiel A, Wiest R, Horn H (2011) Semantic memory involvement in the default mode network: a functional neuroimaging study using independent component analysis. Neuroimage 54:3057-3066.

Wong PCM, Jin JX, Gunasekera GM, Abel R, Lee ER, Dhar S (2009) Aging and cortical mechanisms of speech perception in noise. Neuropsychologia 47:693-703.

Wong PCM, Uppunda AK, Parrish TB, Dhar S (2008) Cortical mechanisms of speech 
1756 perception in noise. J Speech Lang Hear Res 51:1026-1041.

1757 Yao Y, Vehtari A, Simpson D, Gelman A (2018) Using stacking to average Bayesian predictive distributions (with discussion). Bayesian Anal Available at: https://projecteuclid.org/euclid.ba/1516093227.

1760

Zekveld AA, Heslenfeld DJ, Festen JM, Schoonhoven R (2006) Top-down and bottom-up processes in speech comprehension. Neuroimage 32:1826-1836. 


\section{Supplementary Information}

The data presented in this study were collected as part of a larger study examining the effects of aging and blast exposure (close proximity to a high-intensity explosion or blast) on auditory processing. As such, the participants were characterized along a number of dimensions including audiological, speech-in-noise, auditory-temporal, mental health, and cognitive measures. Supplementary Table 1 provides a summary of these measures including a brief description and relevant references. Here, we provide an abbreviated description of the methods used to collect these measures and a description of how the data were preprocessed for entry into principal component analysis as described in Section 2.3.2 of the main manuscript.

\section{Supplementary Table 1}

\begin{tabular}{|c|c|c|c|}
\hline Measure & Type & Description & References \\
\hline Age & $\mathrm{n} / \mathrm{a}$ & Age in years at time of enrollment. & n/a \\
\hline $\begin{array}{l}\text { High-Freq } \\
\text { Average } \\
\text { Thresholds }\end{array}$ & Audiological & $\begin{array}{l}\text { Average of pure tone, air conduction threhsolds in the better } \\
\text { ear at audiometric frequencies from } 2-6 \mathrm{kHz}(\mathrm{dB} \mathrm{HL}) \text {. }\end{array}$ & n/a \\
\hline $\begin{array}{l}\text { Speech-shaped } \\
\text { noise threshold }\end{array}$ & Audiological & $\begin{array}{l}\text { Threshold for detection of speech spectrum noise in the } \\
\text { better ear ( } d B \mathrm{HL}) \text {. }\end{array}$ & $n / a$ \\
\hline $500 \mathrm{~Hz}$ MLD & Audiological & $\begin{array}{l}\text { Threshold ( } \mathrm{dB} \mathrm{HL} \text { ) for detection of a } 500 \mathrm{~Hz} \text { tone in } \\
\text { background noise where the tone and noise are in phase } \\
\text { between the ears (NOSO) and where the noise is in phase } \\
\text { but the tone is out of phase between the ears (NOSpi). Only } \\
\text { NOSpi used in further analysis. }\end{array}$ & $\begin{array}{l}\text { (Bernstein \& Trahiotis, } \\
\text { 1999) }\end{array}$ \\
\hline $\begin{array}{l}\text { Gap Duration } \\
\text { Discrimination } \\
\text { (GDD) }\end{array}$ & $\begin{array}{l}\text { Temporal } \\
\text { Processing }\end{array}$ & $\begin{array}{l}\text { AXB discrimination. Standard interval }=432 \mathrm{~Hz} \text { tone, silent } \\
\text { gap, } 458 \mathrm{~Hz} \text { tone. Signal interval }=432 \mathrm{~Hz} \text { tone, silent gap }+ \\
\text { DELTA, } 458 \mathrm{~Hz} \text { tone. Tones are } 20-\mathrm{ms} \text { in duration with } \\
10-\mathrm{ms} \text { cosine squared on/off ramps. DELTA is adapted using } \\
\text { a weighted up-down procedure to target } 77.85 \% \text { correct. A } \\
\text { single run with } 40 \text { reversals is obtained. The step size is } \\
\text { reduced after the } 2 \mathrm{nd} \text { and } 4 \text { th reversals. Threshold is the } \\
\text { geometric mean of DELTA (ms) at the final } 36 \text { reversals. Two } \\
\text { conditions: standard gap }=0 \mathrm{~ms} \text {, standard gap = } 35 \mathrm{~ms} \text {. } \\
\text { Monaural, } 80 \mathrm{~dB} \text { SPL. }\end{array}$ & $\begin{array}{l}\text { (García-Pérez, 2011; } \\
\text { Grose et al., 2006) }\end{array}$ \\
\hline $\begin{array}{l}\text { Frequency } \\
\text { Modulation } \\
\text { Detection }\end{array}$ & $\begin{array}{l}\text { Temporal } \\
\text { Processing }\end{array}$ & $\begin{array}{l}\text { AXB discrimination. Standard interval }=1250-\mathrm{ms} \text { pure tone. } \\
\text { Signal interval = } 1250-\mathrm{ms} \text { tone with } 2 \mathrm{~Hz} \text { frequecy } \\
\text { modulation (FM). Carrier frequency roved from } 460-540 \mathrm{~Hz} \\
\text { from interval to interval (random starting phase). FM depth is } \\
\text { adapted according to a weighted up-down staricase as } \\
\text { described for GDD. Threshold obtained as the geometric } \\
\text { mean FM depth }(\mathrm{Hz}) \text { at the final } 36 \text { reversals. Monaural, } 75 \\
\text { dB SPL. }\end{array}$ & (Grose \& Mamo, 2012) \\
\hline $\begin{array}{l}\text { Forward } \\
\text { Masking }\end{array}$ & $\begin{array}{l}\text { Temporal } \\
\text { Processing }\end{array}$ & $\begin{array}{l}\text { AXB discrimination. Standard interval = 400-ms segment of } \\
\text { speech spectrum noise at } 80 \mathrm{~dB} \text { SPL transitions to } 45 \mathrm{~dB} \\
\text { SPL. Signal interval = same as standard, except that a } \\
30-\mathrm{ms} \text { segment speech spectrum noise at LEVEL (dB SPL) } \\
\text { is added to the standard following a GAP between the initial } \\
400-\mathrm{ms} \text { noise segment and the } 30 \text {-ms noise segment. } \\
\text { LEVEL is adapted according to a weighted up-down } \\
\text { staircase as described for GDD. Threshold obtained as the } \\
\text { mean LEVEL at the final } 36 \text { reversals. Two conditions: GAP }\end{array}$ & (Grose et al., 2016) \\
\hline
\end{tabular}




\begin{tabular}{|c|c|c|c|}
\hline & & $=16 \mathrm{~ms}, \mathrm{GAP}=128 \mathrm{~ms}$. Monaural. & \\
\hline $\begin{array}{l}\text { Interaural } \\
\text { Phase-Differenc } \\
\text { e Detection }\end{array}$ & $\begin{array}{l}\text { Temporal } \\
\text { Processing }\end{array}$ & $\begin{array}{l}\text { AXB discrimination. Standard interval (diotic) }=800-m s \text { pure } \\
\text { tone ( } 75 \mathrm{~dB} \text { SPL) amplitude modulated at } 5 \mathrm{~Hz}(100 \% \\
\text { depth); the initial carrier phase is }+45 \text { degrees, but phase } \\
\text { changes to }-45 \text { degrees at the first minimum in the AM cycle, } \\
\text { then back to }+45 \text { at the next minimum in the AM cycle, etc., } \\
\text { throughout the stimulus. Signal (dichotic) = same as } \\
\text { standard, but the carrier phases changed from }+45 /-45 \\
\text { degrees in the left/right channels to }-45 /+45 \text { degrees at the } \\
\text { first minimum, etc. ( } 180 \text { degree interaural phase } \\
\text { modulation). The carrier frequency (FREQ) was adapted } \\
\text { according to a weighted up-down staircase as described for } \\
\text { GDD (higher FREQ = more difficult). Threshold was obtained } \\
\text { as the geometric mean FREQ at the final } 36 \text { reversals. }\end{array}$ & \begin{tabular}{|l} 
\\
\\
(Grose and Mamo 2010; \\
Undurraga et al. 2016)
\end{tabular} \\
\hline $\begin{array}{l}\text { Words in Noise } \\
\text { Test (WIN) }\end{array}$ & $\begin{array}{l}\text { Speech in } \\
\text { Noise }\end{array}$ & $\begin{array}{l}\text { Word recognition in multitalker babble at seven } \\
\text { signal-to-noise ratios. Outcome is the } 50 \% \text { correct point (dB } \\
\text { SNR) calculated using the Spearman-Karber equation. } \\
\text { Average over two } 35 \text {-word lists. }\end{array}$ & (Wilson 2011) \\
\hline QuickSIN & $\begin{array}{l}\text { Speech in } \\
\text { Noise }\end{array}$ & $\begin{array}{l}\text { The quick speech-in-noise test. Sentence } \\
\text { keyword-recognition in multitalker babble at six different } \\
\text { signal-to-nosie ratios. The outcome is the } 50 \% \text { correct point } \\
\text { (dB SNR) relative to the average normal hearing listner (as } \\
\text { dB SNR Loss). }\end{array}$ & (Killion et al. 2004) \\
\hline TVM & $\begin{array}{l}\text { Competing } \\
\text { Speech }\end{array}$ & $\begin{array}{l}\text { Modified version of the Theo-Victor-Michael competing } \\
\text { speech test. Target speech was presented diotically at } 80 \mathrm{~dB} \\
\text { SPL. Four background noise conditions, each at } 0 \mathrm{~dB} \\
\text { signal-to-noise ratio: speech spectrum noise, } \\
\text { speech-envelope modulated speech spectrum noise, one } \\
\text { competing talker, two competing talkers. A measure of } \\
\text { competing speech interference was obtained as the average } \\
\text { proportion correct performance (as log odds) in the two } \\
\text { speech spectrum noise conditions minus average proportion } \\
\text { correct performance (as log odds) in the two competing } \\
\text { speech conditions. }\end{array}$ & $\begin{array}{l}\text { |Helfer \& Freyman, } \\
\text { (2009) }\end{array}$ \\
\hline SSQ6 & $\begin{array}{l}\text { Subjective } \\
\text { Hearing }\end{array}$ & $\begin{array}{l}\text { Six item subset of the Speech, Spatial, and Qualities } \\
\text { questionnaire (total score). }\end{array}$ & $\begin{array}{l}\text { (Shinn-Cunningham et } \\
\text { al., 2019) }\end{array}$ \\
\hline HHIA & $\begin{array}{l}\text { Subjective } \\
\text { Hearing }\end{array}$ & Hearing Handicap Inventory for Adults (total score). & (Newman et al. 1990) \\
\hline CES-D & $\begin{array}{l}\text { Mental } \\
\text { Health }\end{array}$ & $\begin{array}{l}\text { Center for Epidemiologic Studies Depression Scale (total } \\
\text { score). }\end{array}$ & (Radloff 1977) \\
\hline PCL-M & $\begin{array}{l}\text { Mental } \\
\text { Health }\end{array}$ & PTSD Checklist Military Version (total score). & (Wilkins et al. 2011) \\
\hline MAST & $\begin{array}{l}\text { Substance } \\
\text { Use }\end{array}$ & Michigan Alcohol Screening Test (total score). & (Skinner \& Sheu, 1982) \\
\hline AMNART & Cognition & $\begin{array}{l}\text { American version of the National Adult Reading Test } \\
\text { (estimated premorbid IQ). }\end{array}$ & (Grober et al. 1991) \\
\hline $\begin{array}{l}\text { WAIS-IV Digit } \\
\text { Span }\end{array}$ & Cognition & $\begin{array}{l}\text { The forward, backward, and sequencing trials of the } \\
\text { Wechsler Adult Intelligence Scale, 4th Edition (WAIS-IV) } \\
\text { Digit Span subtest. }\end{array}$ & (Wechsler 2008) \\
\hline
\end{tabular}




\begin{tabular}{l|l|l|l|}
\hline SDMT & Cognition & Symbol Digit Modalities Test (total hits). & (Smith 1968) \\
\hline Stroop Test & Cognition & $\begin{array}{l}\text { The Word, Color, and Color-Word trialss of the Stroop test } \\
\text { (total correct items). }\end{array}$ & (Golden et al. 1978) \\
\hline $\begin{array}{l}\text { Letter } \\
\text { Cancellation }\end{array}$ & Cognition & $\begin{array}{l}\text { The "Cancel H" Letter Cancellation test (total hits and } \\
\text { completion time). }\end{array}$ & $\begin{array}{l}\text { (Uttl and } \\
\text { Pilkenton-Taylor 2001) }\end{array}$ \\
\hline $\begin{array}{l}\text { Trail-Making } \\
\text { Test }\end{array}$ & Cognition & Parts A \& B of the Trail Making Test (completion time). & (Tombaugh 2004) \\
\hline FAS Test & Cognition & $\begin{array}{l}\text { FAS (phonemic) verbal fluency test (total number of words } \\
\text { generated). }\end{array}$ & (Tombaugh et al. 1999) \\
\hline $\begin{array}{l}\text { WAIS-IV } \\
\text { Cancellation }\end{array}$ & Cognition & WAIS-IV Cancellation subtest (total score). & (Wechsler 2008) \\
\hline $\begin{array}{l}\text { NIH Toolbox } \\
\text { Cognition } \\
\text { Battery }\end{array}$ & Cognition & $\begin{array}{l}\text { The Flanker Inhibitory Control, List Sorting Working Memory, } \\
\text { Dimensional Change Card Sort, and Pattern Comparison } \\
\text { Processing Speed tests of the NIH Toolbox Cognition Battery } \\
\text { (uncorrected standard scores). }\end{array}$ & (Weintraub et al. 2013) \\
\hline & $\begin{array}{l}\text { Custom implementation of the CogState Brief Detection } \\
\text { (simple reaction time) and Identification (choice reaction } \\
\text { time) tasks (mean logarithm base 10 transformed reaction } \\
\text { time after removing outliers). }\end{array}$ & (Maruff et al., 2009) \\
\hline
\end{tabular}

\section{Abbreviated Methods}

Audiological and speech-in-noise measures were collected on a Madsen Astera2 audiometer (Otometrics, Inc.) with ER-3 insert earphones (Etymotic Research, Inc.) using programs available in the OtoSuite software package. The only exception was speech-shaped noise thresholds, for which a custom speech spectrum noise signal was generated to match the long-term average speech spectrum of the four female talkers in the Theo-Victor-Michael stimulus set (Helfer \& Freyman, 2009); obtained with permission from the authors). Two hundred 5-s segments of speech spectrum noise were generated, uploaded to the Astera2 audiometer, and the audiometer dial was set to the 'speech' setting ( $d B \mathrm{HL})$. Thresholds were obtained using the Hughson-Westlake procedure.

Auditory temporal processing measures were obtained using custom MATLAB (The Mathworks, Inc.) programs implemented on a Tucker Davis WS4 PC running Windows 7. Stimulus presentation and timing were controlled via PsychToolbox v3 (Kleiner et al., 2007). Acoustic stimuli were delivered via a Scarlett $2 \mathrm{i} 2$ external sound card with ASIO drivers and ER-2 insert earphones (Etymotic Research, Inc.), with each channel amplified by a separate Magni 2 headphone amplifier (Schiit, Inc.). Visual stimuli were presented on an Elo 1502L 15-in touchscreen monitor (Elo Touch Solutions, Inc.), which was also used to acquire responses from the participants.

The TVM competing speech test was implemented using the same setup as the auditory temporal processing measures. Each item in the TVM has two target keywords, which are one- or two-syllable nouns selected largely from the Thorndike-Lorge list. Participants reported the keywords verbally and an experimenter scored each item in real time using a custom graphical user interface. Participants completed 12 practice trials and 48 experimental trials in each condition.

Unless otherwise stated, cognitive measures were obtained using standard "paper-and-pencil" instruments with manual timing and scoring, either by a trained neuropsychologist or a research audiologist trained by a neuropsychologist to administer the test battery. The NIH Toolbox measures were obtained on an iPad (Apple, 
Inc.) using semi-automated programs available in the NIH Toolbox iPad application. The simple and choice reaction time measures were obtained using custom MATLAB programs with the same setup as the auditory temporal and TVM measures.

\section{Data Preprocessing}

Prior to principal component analysis, some of the measures were first collapsed into composite variables using the average-standard-score technique. That is, each individual measure was z-scored and sign-flipped as appropriate to ensure that positive values corresponded to better performance, and then different subsets of the standardized measures were averaged to produce composite variables as follows: temporal fine structure processing (frequency modulation and interaural phase difference detection), envelope processing (all conditions of gap duration discrimination and forward masking), mental health (CES-D and PCL-M), selective attention (Letter Cancellation, WAIS-IV Cancellation, Stroop Color-Word, NIH Toolbox Flanker, choice reaction time), executive function (FAS, Trails B, Digit Span Backward and Sequencing, NIH Toolbox List Sort, NIH Toolbox Card Sort), and attention/processing speed (Digit Span Forward, SDMT, Trails A, Stroop Color, Stroop Word, NIH Toolbox Pattern Comparison, simple reaction time). The composite measures were then re-standardized (z-scored) and the remaining measures were also z-scored prior to principal component analysis.

\section{References}

Bernstein, L. R., \& Trahiotis, C. (1999). The effects of signal duration on NoSo and NoS pi thresholds at 500 $\mathrm{Hz}$ and $4 \mathrm{kHz}$. The Journal of the Acoustical Society of America, 105(3), 1776-1783.

García-Pérez, M. A. (2011). A cautionary note on the use of the adaptive up-down method. The Journal of the Acoustical Society of America, 130(4), 2098-2107.

Golden, C.J., T.A. Hammeke, and A.D. Purisch, Diagnostic validity of a standardized neuropsychological battery derived from Luria's neuropsychological tests. J Consult Clin Psychol, 1978. 46(6): p. 1258-65.

Grober, E., Sliwinsk, M., \& Korey, S. R. (1991). Development and validation of a model for estimating premorbid verbal intelligence in the elderly. Journal of Clinical and Experimental Neuropsychology, 13(6), 933-949.

Grose, J. H., Hall, J. W., lii, \& Buss, E. (2006). Temporal processing deficits in the pre-senescent auditory system. The Journal of the Acoustical Society of America, 119(4), 2305-2315.

Grose, J. H., \& Mamo, S. K. (2010). Processing of temporal fine structure as a function of age. Ear and Hearing, 31(6), 755.

Grose, J. H., \& Mamo, S. K. (2012). Frequency modulation detection as a measure of temporal processing: Age-related monaural and binaural effects. Hearing Research, 294(1), 49-54.

Grose, J. H., Menezes, D. C., Porter, H. L., \& Griz, S. (2016). Masking period patterns and forward masking for speech-shaped noise: Age-related effects. Ear and Hearing, 37(1), 48-54.

Helfer, K. S., \& Freyman, R. L. (2009). Lexical and indexical cues in masking by competing speech. The Journal of the Acoustical Society of America, 125(1), 447-456.

Killion, M. C., Niquette, P. A., Gudmundsen, G. I., Revit, L. J., \& Banerjee, S. (2004). Development of a quick speech-in-noise test for measuring signal-to-noise ratio loss in normal-hearing and hearing-impaired listeners. The Journal of the Acoustical Society of America, 116(4), 2395-2405.

Kleiner, M., Brainard, D., Pelli, D., Ingling, A., Murray, R., \& Broussard, C. (2007). What's new in Psychtoolbox-3. Perception, 36(14), 1.1-16.

Maruff, P., Thomas, E., Cysique, L., Brew, B., Collie, A., Snyder, P., \& Pietrzak, R. H. (2009). Validity of the CogState brief battery: relationship to standardized tests and sensitivity to cognitive impairment in mild traumatic brain injury, schizophrenia, and AIDS dementia complex. Archives of Clinical Neuropsychology: 
The Official Journal of the National Academy of Neuropsychologists, 24(2), 165-178.

Newman, C. W., Weinstein, B. E., Jacobson, G. P., \& Hug, G. A. (1990). The Hearing Handicap Inventory for Adults: psychometric adequacy and audiometric correlates. Ear and Hearing, 11(6), 430-433.

Radloff, L. S. (1977). The CES-D Scale: A Self-Report Depression Scale for Research in the General Population. Applied Psychological Measurement, 1(3), 385-401.

Shinn-Cunningham, B., Grant, K., \& Bressler, S. (2019). Diagnosing Contributions of Sensory and Cognitive Deficits to Hearing Dysfunction in Blast-Exposed/TBI Service Members. Trustees of Boston University Boston United States. https://apps.dtic.mil/sti/pdfs/AD1094820.pdf

Skinner, H. A., \& Sheu, W.-J. (1982). Reliability of alcohol use indices. The Lifetime Drinking History and the MAST. Journal of Studies on Alcohol, 43(11), 1157-1170.

Smith, A. (1968). The symbol-digit modalities test: a neuropsychologic test of learning and other cerebral disorders. Learning Disorders, 83-91.

Tombaugh, T. N. (2004). Trail Making Test A and B: normative data stratified by age and education. Archives of Clinical Neuropsychology: The Official Journal of the National Academy of Neuropsychologists, 19(2), 203-214.

Tombaugh, T. N., Kozak, J., \& Rees, L. (1999). Normative data stratified by age and education for two measures of verbal fluency: FAS and animal naming. Archives of Clinical Neuropsychology: The Official Journal of the National Academy of Neuropsychologists, 14(2), 167-177.

Undurraga, J. A., Haywood, N. R., Marquardt, T., \& McAlpine, D. (2016). Neural Representation of Interaural Time Differences in Humans-an Objective Measure that Matches Behavioural Performance. Journal of the Association for Research in Otolaryngology: JARO, 17(6), 591-607.

Uttl, B., \& Pilkenton-Taylor, C. (2001). Letter cancellation performance across the adult life span. The Clinical Neuropsychologist, 15(4), 521-530.

Wechsler, D. (2008). Wechsler Adult Intelligence Scale-Fourth Edition (WAIS-IV).

Weintraub, S., Dikmen, S. S., Heaton, R. K., Tulsky, D. S., Zelazo, P. D., Bauer, P. J., Carlozzi, N. E., Slotkin, J., Blitz, D., Wallner-Allen, K., Fox, N. A., Beaumont, J. L., Mungas, D., Nowinski, C. J., Richler, J., Deocampo, J. A., Anderson, J. E., Manly, J. J., Borosh, B., ... Gershon, R. C. (2013). Cognition assessment using the NIH Toolbox. Neurology, 80(11 Suppl 3), S54-S64.

Wilkins, K. C., Lang, A. J., \& Norman, S. B. (2011). Synthesis of the psychometric properties of the PTSD checklist (PCL) military, civilian, and specific versions. Depression and Anxiety, 28(7), 596-606.

Wilson, R. H. (2011). Clinical experience with the words-in-noise test on 3430 veterans: comparisons with pure-tone thresholds and word recognition in quiet. Journal of the American Academy of Audiology, 22(7), 405-423. 